\title{
Methylation-dependent loss of RIP3 expression in cancer represses programmed necrosis in response to chemotherapeutics
}

Gi-Bang Koo ${ }^{1,2, *}$, Michael J Morgan ${ }^{3, *}$, Da-Gyum Lee ${ }^{1}$, Woo-Jung Kim ${ }^{1,2}$, Jung-Ho Yoon ${ }^{1,2}$, Ja Seung Koo ${ }^{4}$ Seung Il Kim ${ }^{5}$, Soo Jung Kim ${ }^{6}$, Mi Kwon Son ${ }^{6}$, Soon Sun Hong ${ }^{6}$, Jean M Mulcahy Levy ${ }^{7}$, Daniel A Pollyea ${ }^{8}$, Craig T Jordan ${ }^{8}$, Pearlly Yan ${ }^{9}$, David Frankhouser ${ }^{9}$, Deedra Nicolet ${ }^{9}, 10$, Kati Maharry ${ }^{9,10}$, Guido Marcucci ${ }^{9}$, Kyeong Sook Choi ${ }^{1,2}$, Hyeseong Cho ${ }^{1,2}$, Andrew Thorburn ${ }^{3}$, You-Sun Kim ${ }^{1,2}$

${ }^{I}$ Department of Biochemistry, Ajou University School of Medicine, Suwon 443-380, Korea; ${ }^{2}$ Department of Biomedical Sciences, Graduate School, Ajou University, Suwon 443-380, Korea; ${ }^{3}$ Department of Pharmacology, University of Colorado School of Medicine, Aurora, CO 80045, USA; ${ }^{4}$ Department of Pathology, Yonsei University College of Medicine, Seoul 120-752, Korea; ${ }^{5}$ Department of Surgery, Yonsei University College of Medicine, Seoul 120-752, Korea; ${ }^{6}$ Department of Biomedical Sciences, College of Medicine, Inha University, Incheon 402-751, Korea; ${ }^{7}$ Department of Pediatrics, University of Colorado Denver, Aurora, CO 80045, USA; ${ }^{8}$ Division of Hematology, Hematologic Malignancies and Stem Cell Transplantation, University of Colorado School of Medicine, Aurora, CO 80045, USA; ${ }^{9}$ Ohio State University Comprehensive Cancer Center, Columbus, OH 43210, USA; ${ }^{10}$ Alliance for Clinical Trials in Oncology Statistics and Data Center, Mayo Clinic, Rochester, MN 55905, USA

Receptor-interacting protein kinase-3 (RIP3 or RIPK3) is an essential part of the cellular machinery that executes "programmed" or "regulated" necrosis. Here we show that programmed necrosis is activated in response to many chemotherapeutic agents and contributes to chemotherapy-induced cell death. However, we show that RIP3 expression is often silenced in cancer cells due to genomic methylation near its transcriptional start site, thus RIP3-dependent activation of MLKL and downstream programmed necrosis during chemotherapeutic death is largely repressed. Nevertheless, treatment with hypomethylating agents restores RIP3 expression, and thereby promotes sensitivity to chemotherapeutics in a RIP3-dependent manner. RIP3 expression is reduced in tumors compared to normal tissue in $85 \%$ of breast cancer patients, suggesting that RIP3 deficiency is positively selected during tumor growth/development. Since hypomethylating agents are reasonably well-tolerated in patients, we propose that RIP3-deficient cancer patients may benefit from receiving hypomethylating agents to induce RIP3 expression prior to treatment with conventional chemotherapeutics.

Keywords: RIP3 (RIPK3); MLKL; programmed necrosis; chemotherapy; hypomethylating agents Cell Research (2015) 25:707-725. doi:10.1038/cr.2015.56; published online 8 May 2015

\section{Introduction}

Resistance to cell death is one hallmark of cancer cells, and tumor cells have developed a variety of strat-

*These two authors contributed equally to this work.

Correspondence: You-Sun Kim

Tel: 82-31-219-4509; Fax: 82-31-219-4530

E-mail: yousunkim@ajou.ac.kr

Received 10 October 2014; revised 8 February 2015; accepted 25 March

2015; published online 8 May 2015 egies to evade cell death [1]. Approaches that overcome such resistance to cell death would therefore be beneficial in cancer therapy and treatment, and allow previously developed chemotherapeutic drugs to be used more effectively. Many previously used stratagems have focused on restoring the ability of a cell to undergo apoptosis. However, other forms of cell death also exist.

The "programmed" or "regulated" necrotic cell death pathway is activated in response to death receptor ligands and other types of cellular stress [2, 3]. Programmed necrotic cell death is distinguished from its counterpart, apoptosis, in that caspase activation is dispensable for 
cell death, and, unlike apoptosis, programmed necrosis results in plasma membrane rupture, thus spilling the contents of the cell and triggering the immune system and inflammation $[3,4]$.

The acceptance of the physiological and pathological relevance of programmed necrosis has come recently due to the recognition that carefully orchestrated repression of the programmed necrotic pathway by apoptotic regulatory proteins, such as FADD and caspase- 8 , is essential for proper mammalian development [5-7], and also prevents spontaneous cell death and inflammation in lymphocytes [6-8], keratinocytes [9], and intestinal epithelial cells $[10,11]$. In addition, programmed necrosis is now acknowledged to play roles in pathological processes [3, $4,12]$, including a facilitative role in tissue damage, such as ischemia-reperfusion injury [13], and in host defense of viral infections [14].

Programmed cell death can be induced by several stimuli, including the proinflammatory cytokine TNF- $\alpha$. Studies of this mode of cell death often utilize the murine fibrosarcoma cell line L929, or the human colon adenocarcinoma cell line HT-29 as models. In the case of L929 cells, TNF- $\alpha$ treatment alone is sufficient to trigger programmed necrosis, while in the case of HT-29 cell line, the addition of either SMAC mimetic or cycloheximide, in combination with the pancaspase inhibitor $\mathrm{zVAD}$, are necessary to induce this type of death.

Receptor-interacting protein kinase-3 (RIP3 or RIPK3) is an essential part of the cellular machinery that executes the programmed necrotic cell death [15-17]. Activation of the canonical programmed necrosis includes the formation of a complex containing RIP3 and RIP1 (RIPK1) and recruitment of mixed lineage kinase domain-like protein (MLKL) [18, 19], which is essential for death to proceed [20, 21]. RIP3-dependent plasma membrane localization of MLKL is necessary for programmed necrotic cell death, and leads to plasma membrane disruption either indirectly $[22,23]$ or directly $[24,25]$.

Here we show that effective cytotoxic chemotherapy is facilitated by RIP3-dependent programmed necrotic cell death; however, RIP3 expression is often silenced in cancer due to genomic methylation near the RIPK3 transcription start site (TSS). We show that a majority of cancer cell lines lack RIP3 expression due to this silencing mechanism, and loss of RIP3 expression in these cell lines leads to greater resistance not only to death receptor ligands, but also to a surprising diversity of standard chemotherapeutic agents, such as DNA-damaging agents and taxanes. Treatment of cells with hypomethylating agents restores RIP3 expression and thereby promotes sensitivity to chemotherapeutics in a RIP3-dependent manner. Lastly, in $>85 \%$ of breast cancer patients, RIP3 expression is reduced in cancer tissue samples compared to normal breast tissue from the same patients, suggesting that deficiency of RIP3 in tumor cells is positively selected during tumor development and/or growth. Since hypomethylating agents are reasonably well-tolerated in patients, an implication of our study is that RIP3-deficient cancer patients may benefit from receiving hypomethylating agents to induce RIP3 expression prior to treatment with conventional chemotherapeutic agents.

\section{Results}

\section{RIP3 contributes to chemosensitivity}

RIP3 is essential for programmed necrosis [15-17]. Consistent with the literature, cells lacking RIP3 expression are completely resistant to prototypical programmed necrotic stimuli (TNF- $\alpha+$ zVAD + either cycloheximide or SMAC mimetic; hereafter referred to as TCZ or TSZ), but become sensitive when RIP3 is ectopically expressed (Supplementary information, Figure S1A), while cells endogenously expressing RIP3 lose their sensitivity to necrotic stimuli when RIP3 is knocked down (Supplementary information, Figure S1B-S1D). RIP3 kinase activity is essential for TNF-induced necrosis (Supplementary information, Figure S1E).

Except a possible contribution to caspase activation downstream of etoposide [26], a role for RIP3 in cell death induced by standard chemotherapeutic cytotoxic agents has never been reported. In HeLa, MDAMB231, and Huh-7 cells (which lack endogenous RIP3 expression), the ectopic expression of RIP3 bestowed additional sensitivity both to etoposide and doxorubicin as measured by multiple assays (Figure 1A and Supplementary information, Figure S2A and S2B). Conversely, in HT-29 cells, which have endogenous RIP3 expression, knockdown of RIP3 inhibited doxorubicin and etoposide cytotoxicity (Figure 1B and Supplementary information, Figure S2C). Surprisingly, ectopic RIP3 expression also increased sensitivity to paclitaxel, camptothecin (CPT), cisplatin, and 5-fluorouracil (5-FU) in multiple cell types (Figure 1C and data not shown). Taken together, these data suggested that RIP3 contributes to the cytotoxicity of multiple drugs with diverse mechanisms of action.

\section{DNA-damaging agents activate RIP3-dependent programmed necrotic cell death}

We sought to investigate the mechanism by which cells were sensitized to chemotherapeutics by RIP3. We first examined whether RIP3 was in the same complex as caspase- 8 upon treatment of cells with etoposide and doxorubicin. These agents led to interaction of caspase- 8 with RIP1 and RIP3, along with FADD, though no in- 
A
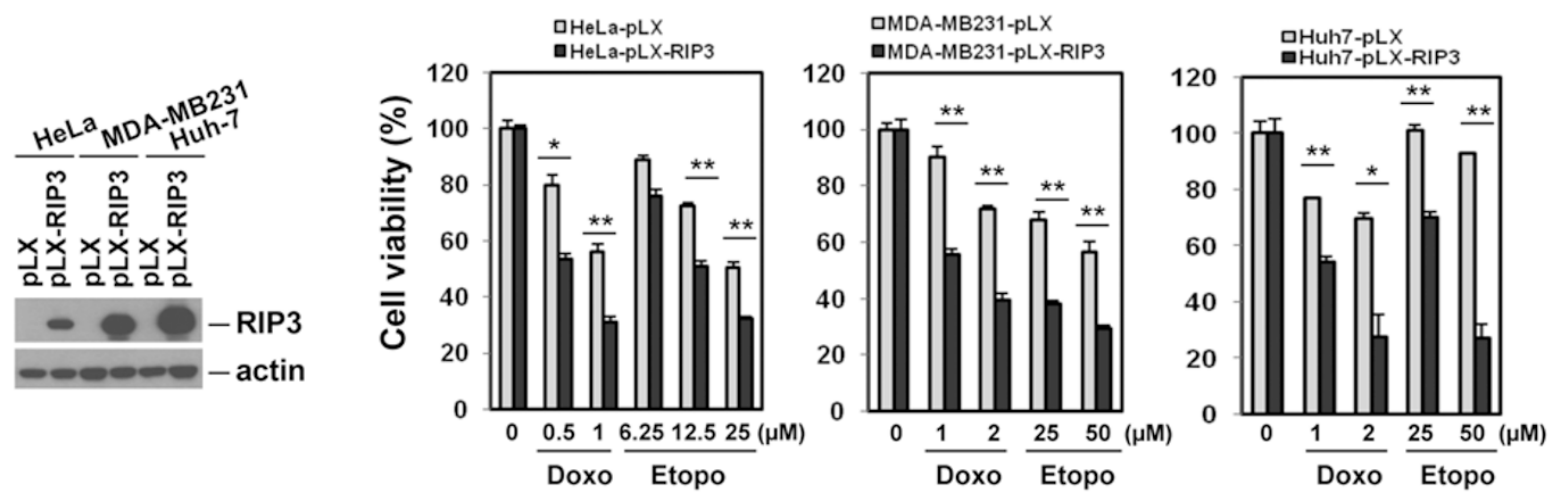

B

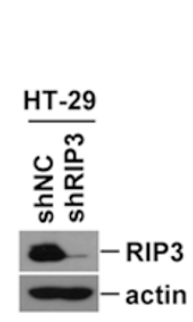

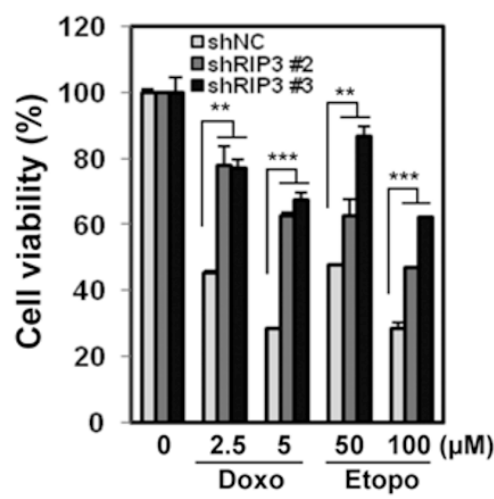

D

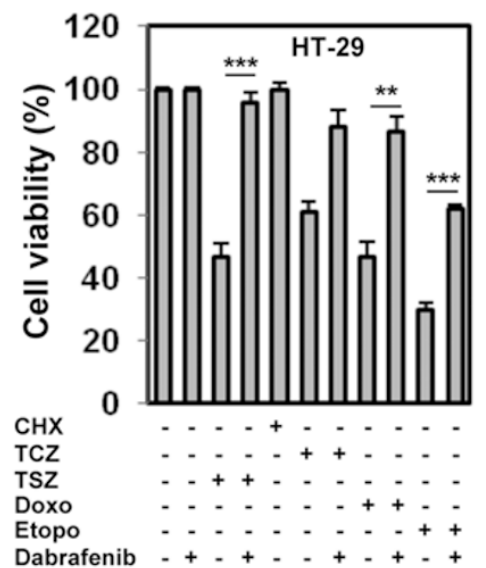

C

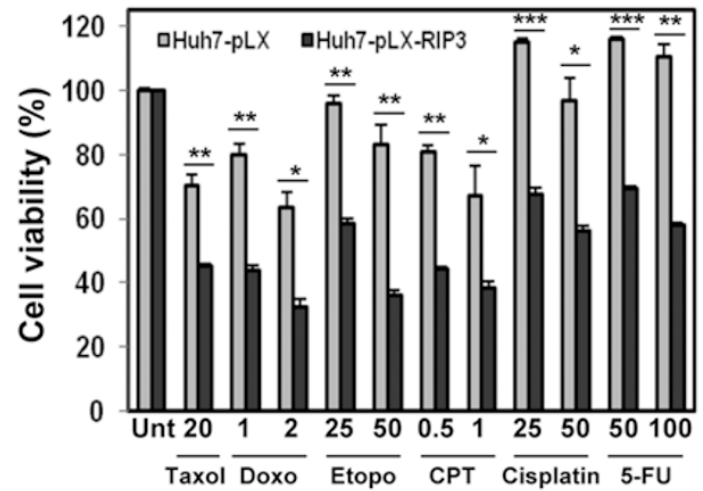

E

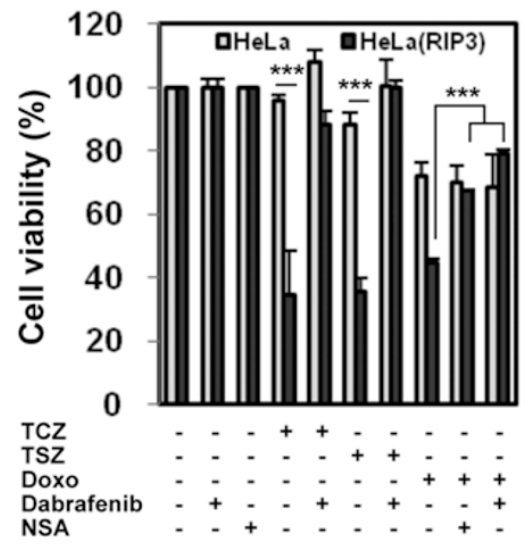

Figure 1 Expression of RIP3 contributes to sensitivity to DNA-damaging agents. (A) HeLa, MDA-MB231, and Huh7 cells ectopically expressing RIP3 were treated with the indicated concentration of doxorubicin or etoposide for 2 days and cell viability was analyzed by MTT assay (right). Results are presented as means \pm SEM. (B) HT-29 cells with stable shRNA expression were analyzed by western blotting (left). Cells were treated with doxorubicin or etoposide for $48 \mathrm{~h}$ and cell viability was analyzed by MTT assay (right). Results are presented as means \pm SEM. (C) Huh7 cells from A were treated with doxorubicin (Doxo, $\mu \mathrm{M})$, etoposide (Etopo, $\mu \mathrm{M})$, taxol $(\mathrm{nM})$, 5-fluorouracil (5-FU, $\mu \mathrm{M})$, camptothecin (CPT, $\mu \mathrm{g} / \mathrm{ml})$, or cisplatin $(\mu \mathrm{M})$, as indicated, and after $48 \mathrm{~h}$, cell viability was analyzed by MTT assay. Results are presented as means \pm SEM. (D) HT-29 cells were pretreated with the RIP3 inhibitor dabrafenib $(10 \mu \mathrm{M})$ for $1 \mathrm{~h}$ and then treated with TCZ/TSZ, doxorubicin, or etoposide for $48 \mathrm{~h}$. Cell viability was analyzed by MTT assay. Results are presented as means \pm SEM. (E) Inhibition of downstream event of RIP3 kinase activation affects cell death in response to DNA-damaging agents. HeLa and RIP3expressing HeLa cells were pretreated with dabrafenib $(10 \mu \mathrm{M})$ or NSA $(1 \mu \mathrm{M})$ for $1 \mathrm{~h}$ and then treated with TCZ/TSZ or doxorubicin $(1 \mu \mathrm{M})$ for $24 \mathrm{~h}$. Cell viability was analyzed by MTT assay. Results are presented as means \pm SEM. ${ }^{*} P<0.05$, ${ }^{* *} P<0.01,{ }^{* *} P<0.001$. 
teraction was detected in untreated cells (Supplementary information, Figure S2D). Unexpectedly, TRADD, a component of TNF-R1 signaling, was also found in the complex (Supplementary information, Figure S2D). We therefore investigated whether autocrine production of TNF- $\alpha$ contributed to cell death. However, an antagonistic TNF-R1 antibody had no effect on doxorubicin-induced cell death despite its ability to prevent TNF-R1-stimulated I $\kappa \mathrm{B} \alpha$ degradation, JNK activation, and cell death (Supplementary information, Figure S2E). In addition, knocking down TNF-R1 by shRNA did not reduce toxicity in response to doxorubicin, though it reduced TSZ-induced cell death (Supplementary information, Figure S2F). Complexes containing RIP1, RIP3, FADD, and caspase- 8 can contribute directly to apoptosis through caspase- 8 activation (ripoptosome) or activate programmed necrosis through RIP3 kinase activity and the phosphorylation of MLKL (necrosome). However, the kinase activity of RIP3 is not thought to contribute to caspase activation $[27,28]$. We therefore sought to determine whether the kinase activity of RIP3 contributes to chemocytotoxicity. The stable ectopic expression of wild-type (WT) RIP3 sensitized the RIP $3^{-/-}$mouse embryonic fibroblasts (MEFs) to doxorubicin and etoposide, while kinase-dead RIP3 failed to do so (Supplementary information, Figure S2G). Thus, while RIP3 is not absolutely required for cell death, RIP3 increases chemosensitivity in a kinase-dependent fashion.

Endogenous RIP3 was observed to interact with endogenous RIP1 in response to doxorubicin (Supplementary information, Figure S3A), and sustained JNK activation, which often accompanies programmed necrosis [29, 30], was elevated in RIP3-expressing cells in response to cisplatin, CPT, and doxorubicin compared to RIP3-deficient cells (Supplementary information, Figure S3B-S3D). Though RIP3 was reported to contribute to caspase activation [26], the above data suggested that programmed necrosis might be important for RIP3-dependent chemocytotoxicity. To further test this hypothesis, we used three pharmacological inhibitors of programmed necrosis, necrostatin-1, necrosulfonamide and dabrafenib. Of these inhibitors, necrostatin-1 inhibits RIP1 kinase activity [31]; dabrafenib inhibits RIP3 kinase activity [32]; while necrosulfonamide inhibits MLKL functions downstream of RIP3 phosphorylation [24]. Necrostatin-1 and necrosulfonamide were substantially better at protecting HT-29 cells from the acute toxicity of etoposide than the pancaspase inhibitor zVAD, which had little effect on cell death (Supplementary information, Figure S4A). Dabrafenib, which fully inhibited TSZ- and TCZ-induced necrosis (Figure 1D and 1E), also inhibited both etoposide- and doxorubicin-induced cell death in HT-29 cells, though to a lesser extent (Figure 1D and Supplementary information, Figure S4B). Likewise, in RIP3-expressing HeLa cells, necrosulfonamide and dabrafenib inhibited doxorubicin- and etoposide-induced cell death (Figure $1 \mathrm{E}$ and Supplementary information, Figure S4C), but had no effect in parental HeLa cells lacking RIP3 expression, suggesting that RIP3 contributed to cell death through programmed necrosis.

MLKL is activated by DNA-damaging agents and is required for RIP3-dependent cell death

In HT-29 cells, MLKL phosphorylation is observed during TNF-induced necrosis (TCZ), but not TNF-induced apoptosis (TC; Supplementary information, Figure S4D), and is inhibited by necrostatin-1 and dabrafenib (Supplementary information, Figure S4E), but not necrosulfonamide ([24], data not shown). In HeLa cells, TSZ induces MLKL phosphorylation only when RIP3 is expressed (Supplementary information, Figure S4F). Importantly, doxorubicin also induced dose- and timedependent MLKL phosphorylation in RIP3-HeLa cells (Figure 2A and 2B), but not in parental HeLa cells (Figure $2 \mathrm{~A}$ and Supplementary information, Figure S4G). However, p53 stabilization was detected in both parental HeLa and RIP3-HeLa cells (Figure 2A and Supplementary information, Figure S4G). Like doxorubicin, etoposide induced MLKL phosphorylation in RIP3-HeLa and HT-29 cells (Figure 2B and Supplementary information, Figure S4H). Phospho-MLKL colocalized with RIP3 in doxorubicin- and etoposide-treated cells, similar to the colocalization pattern seen during TNF-induced necrosis (Figure 2C). Furthermore, ectopic and endogenous RIP3 could be co-immunoprecipitated with MLKL after doxorubicin treatment in HeLa and HT-29 cells, respectively (Figure 2D and 2E, Supplementary information, Figure S4I). Doxorubicin-induced MLKL phosphorylation was inhibited by dabrafenib, but not by necrosulfonamide (Supplementary information, Figure S4J), consistent with their mechanisms of inhibition during TNF-induced necrosis. Significantly, MLKL knockdown in either RIP3-HeLa or HT-29 cells inhibited doxorubicin or etoposide cytotoxicity (Figure $2 \mathrm{~F}$ and 2G, Supplementary information, Figure S4K), suggesting that RIP3 promotes cell death through activation of MLKL-dependent programmed necrosis.

RIP3 is silenced in many cancer cell lines, but not in normal cells, through methylation-dependent mechanisms

While the above data indicate that programmed necrosis contributes to chemotherapeutic cytotoxicity, many cancer cell lines are resistant to programmed necrosis induced by TNF and other stimuli. RIP3 protein is absent 
A

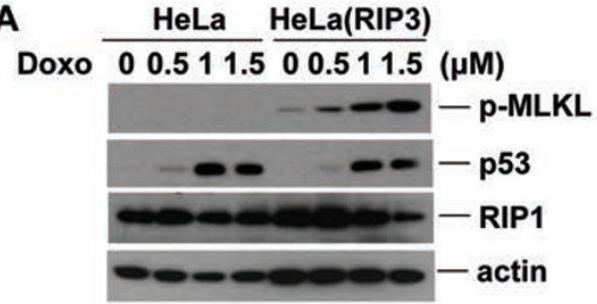

C

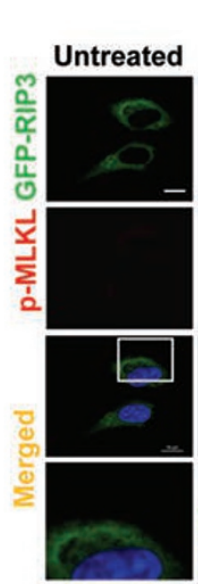

D

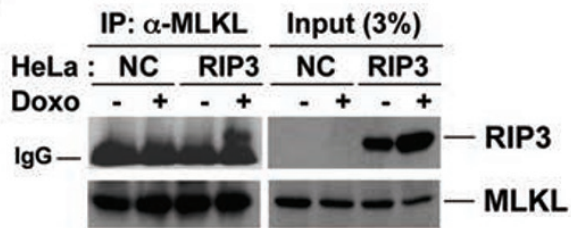

F

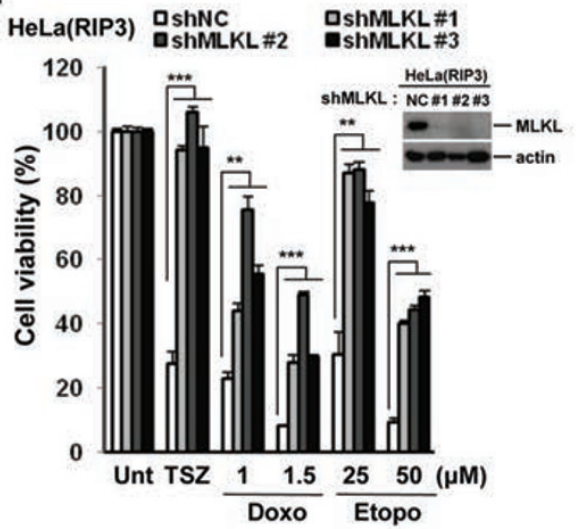

B
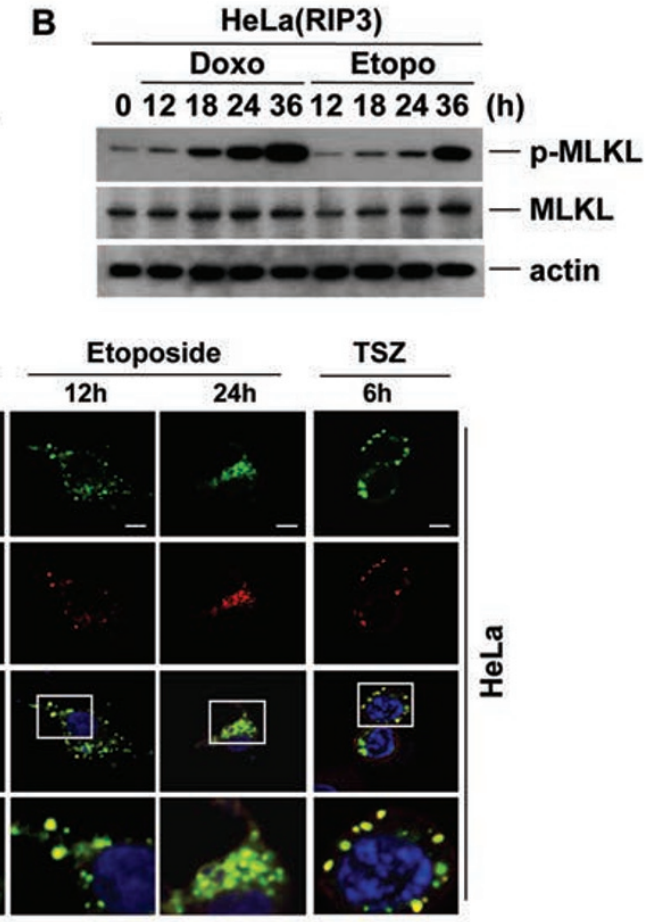

E

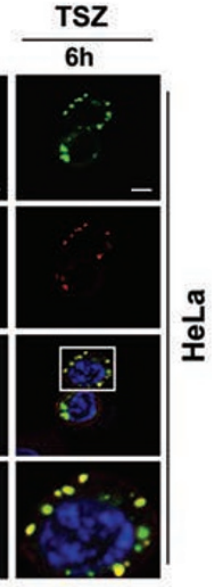

HT-29

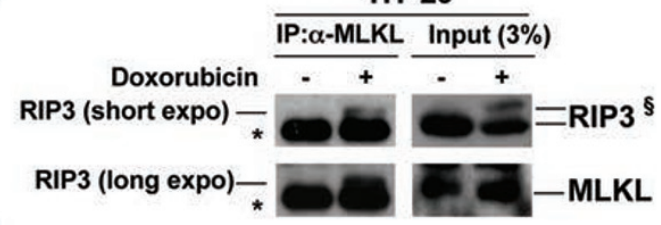

G

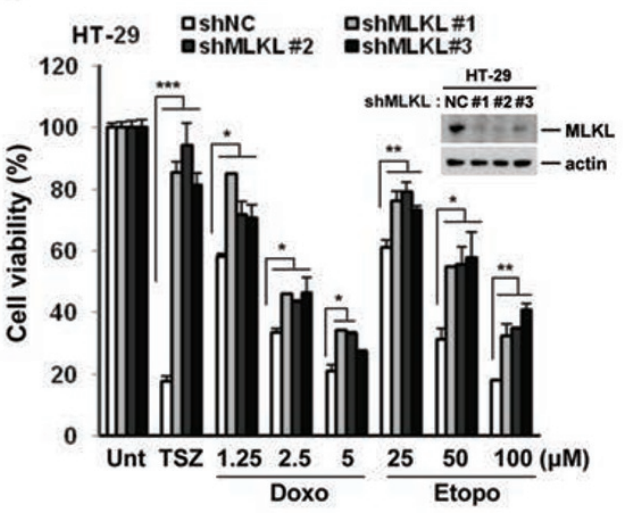

Figure 2 MLKL phosphorylation induced by DNA-damaging agents is dependent on RIP3 kinase activity. (A) HeLa and RIP3-expressing HeLa cells were treated with different concentrations of doxorubicin for $24 \mathrm{~h}$ and cell lysates were analyzed by western blotting. (B) RIP3-expressing HeLa cells were treated with doxorubicin $(0.5 \mu \mathrm{M})$ or etoposide $(12.5 \mu \mathrm{M})$ for the indicated times and cell lysates were analyzed by western blotting. (C) HeLa cells were transiently transfected with GFPhRIP3 and cells were treated with TCZ, doxorubicin $(0.5 \mu \mathrm{M})$, and etoposide $(12.5 \mu \mathrm{M})$ for the indicated times. Cells were stained with phospho-MLKL antibody and analyzed by confocal fluorescence microscopy. Scale bar, $10 \mu \mathrm{m}$. (D) HeLa and RIP3-expressing HeLa cells were treated with doxorubicin $(1 \mu \mathrm{M})$ for $12 \mathrm{~h}$ and cell lysates were immunoprecipitated with anti-MLKL antibody. (E) HT-29 cells were treated with doxorubicin $(1.25 \mu \mathrm{M})$ for $24 \mathrm{~h}$. Cell lysates were immunoprecipitated with anti-MLKL antibody and analyzed by western blotting for RIP3. The asterisk $\left({ }^{*}\right)$ indicates the IgG band. ${ }^{\S}$ The upper band in this blot is thought to be phosphorylated RIP3, and appears only in the doxorubicin-treated cells. (F, G) RIP3-expressing HeLa (F) or HT-29 cells expressing MLKL shRNAs or non-silencing control (G) were analyzed by western blotting (insets). Cells were treated with TSZ, doxorubicin, or etoposide for $48 \mathrm{~h}$ and cell viability was analyzed by MTT assay. Results are presented as means \pm SEM. ${ }^{*} P<0.05,{ }^{* *} P<0.01,{ }^{* * *} P<0.001$. 
in about two-thirds of the 60+ cancer cell lines that we have tested. While only $20 \%$ of examined hematopoetic cell lines lack RIP3 expression, $80 \%$ of the other cell lines have no detectable RIP3 (Figure 3A). In contrast, RIP3 is expressed in primary cells and most normal tissues, as well as in some non-transformed cell lines, as measured by western blotting or immunohistochemical staining in paraffin-embedded specimens (Figure 3B and Supplementary information, S5A and S5B), indicating that RIP3 deficiency could be associated with cancer development or progression. We therefore sought to determine the mechanism(s) responsible for RIP3 deficiency in cancer cells. Protein insolubility did not contribute to lack of RIP3 detection (Figure 3C). Unlike the protein NIK, which is constitutively degraded by the proteosome, inhibition of proteosome function by MG132 did not lead to increased RIP3 (Figure 3D), suggesting that lack of RIP3 expression is not due to constitutive protein turnover.

We examined whether DNA methylation or histone deacetylation was responsible for RIP3 deficiency. HDAC inhibitors TSA and sodium butyrate did not affect RIP3 expression in HeLa cells on their own. However, treatment with the hypomethylating agent decitabine (5-aza-2'-deoxycytidine, abbreviated as 5-AD) restored RIP3 expression (Figure 3E). In multiple cell lines, 5-AD induced RIP3 expression in a time- and dose-dependent manner (Figure 3F). Approximately $70 \%$ of tested cell lines responded to 5-AD by upregulating RIP3 protein (Figure 4A), suggesting that methylation-dependent RIP3 silencing is prevalent. Mouse cancer cell lines also responded to 5-AD by upregulating RIP3 (Supplementary information, Figure S5C and S5D). RIP3 mRNA was largely absent in cells lacking RIP3 protein; however, 5-AD upregulated RIP3 mRNA in a manner similar to sFRP2 (Figure 4B), a protein previously shown to be silenced by promoter methylation [33]. Treatment with 5-AD did not affect RIP3 mRNA or protein in cells already expressing RIP3 (Supplementary information, Figure S5E and S5F). RIP3 mRNA and protein expression were similarly induced by other hypomethylating agents, including 5-azacytidine (5-AZA) and RG108 (Figure 4C and 4D, Supplementary information, Figure S5G and $\mathrm{S} 5 \mathrm{H})$.

Our analysis of RIPK3 methylation from the publicly available databases (such as the University of California-Santa Cruz Genome Browser, among others), combined with analysis of our RIP3 protein expression data, suggested that the correlation between RIP3 silencing and the methylation of a given $\mathrm{CpG}$ is strongest very near the TSS, and is much reduced a few hundred basepairs downstream of the TSS. These databases suggest that a CpG island exists about 240-600 bp downstream of the TSS. Therefore, these CpGs would be considered to be on the "northern $\mathrm{CpG}$ island shore". $\mathrm{CpG}$ island shore methylation has been shown to be sometimes strongly related to gene expression, even more so than the islands themselves [34]. Therefore, we sought to investigate whether methylation of this region was responsible for RIP3 silencing. Not surprisingly, methylation-specific PCR indicated that methylation occurred in this region of genomic DNA near the TSS of RIPK3 in RIP3-silenced cells (MDA-MB231, K562, HeLa), but not in RIP3-expressing HT-29 cells (Figure 4E). Further methylation-specific PCR using multiple primer sets in several different cell lines indicated that the methylation of RIPK3 genomic DNA was responsive to 5-AD (Figure $4 \mathrm{~F}$ ), and methylation status inversely corresponded to mRNA and protein expression in these cell lines under treated and non-treated conditions (compare Figure $4 \mathrm{~F}$ to Figures 3A, 3F, 4A and 4B).

To verify that the expression of RIP3 was associated with methylation of genomic DNA near the TSS of $R I P K 3$, we conducted pyrosequencing of individual CpGs in bisulfate-converted DNA within this region in multiple cell lines treated or untreated with 5-AD (Supplementary information, Figure S6A and S6B). Analysis of these experiments suggested that silencing of RIP3 expression was especially associated with four proximal CpGs just downstream of the RIP $3 K$ TSS. Cells that were resistant to 5-AD in terms of RIP3 reexpression (i.e., MDA-MB468 and A549N) responded minimally in terms of reduction of methylation in any sequenced CpGs. On the other hand, HT-29 cells, which express RIP3 highly, had very low methylation of the entire region.

To examine the role of methyltransferases in RIP3 silencing, we knocked down maintenance methyltransferase, DNMT1. DNMT1 knockdown led to RIP3 mRNA expression (Figure 4G). Furthermore, 5-AD treatment led to the loss of DNMT1 protein (Figure 4H and Supplementary information, Figure S7A), consistent with proteosomal degradation of inhibited DNMT1, which has been previously observed following treatment with hypomethylating agents [35]. In contrast, the expression of DNMT3a protein was only slightly reduced by 5-AD (Supplementary information, Figure S7A). The mRNA of DNMT1 also seemed to be reduced in HeLa and DLD1 cells in the presence of 5-AD; however, DNMT3a and DNMT3b expression was not reduced after 5-AD treatment (Supplementary information, Figure S7B). In addition, HT-29 cells, which express endogenous RIP3, had very little, if any, DNMT1 mRNA (Supplementary information, Figure S7B). RIP3 mRNA was upregulated in response to knockdown of DNMT1; however, the 
A

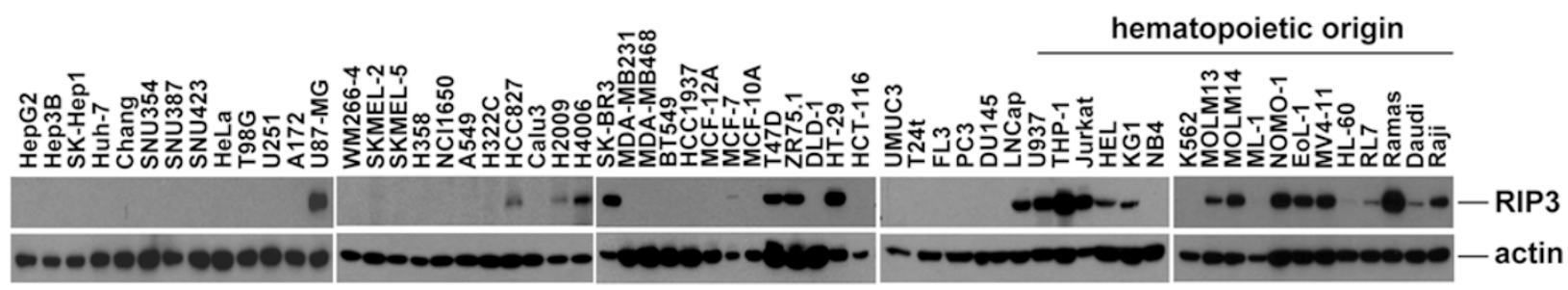

B

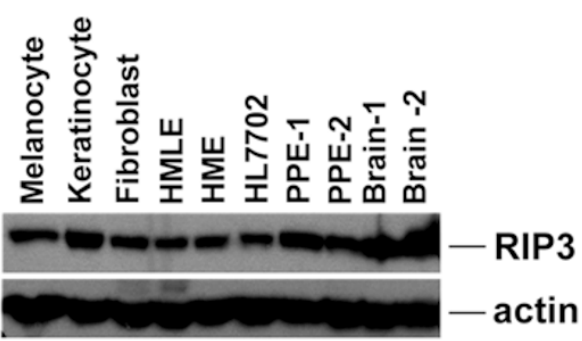

D

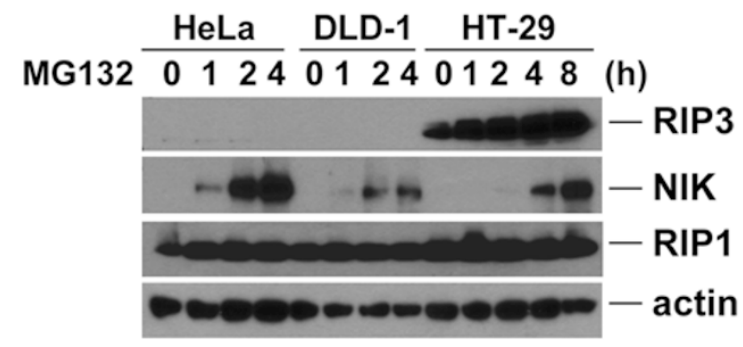

C

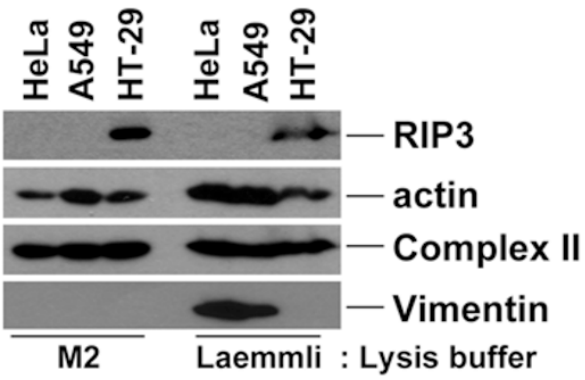

E

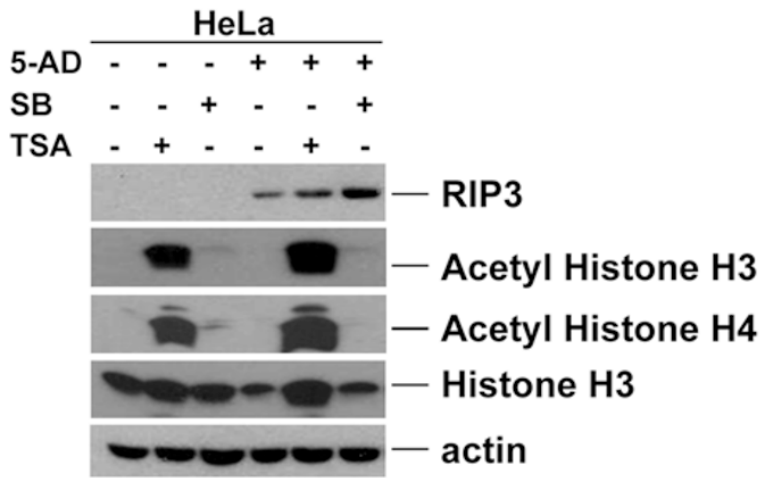

F
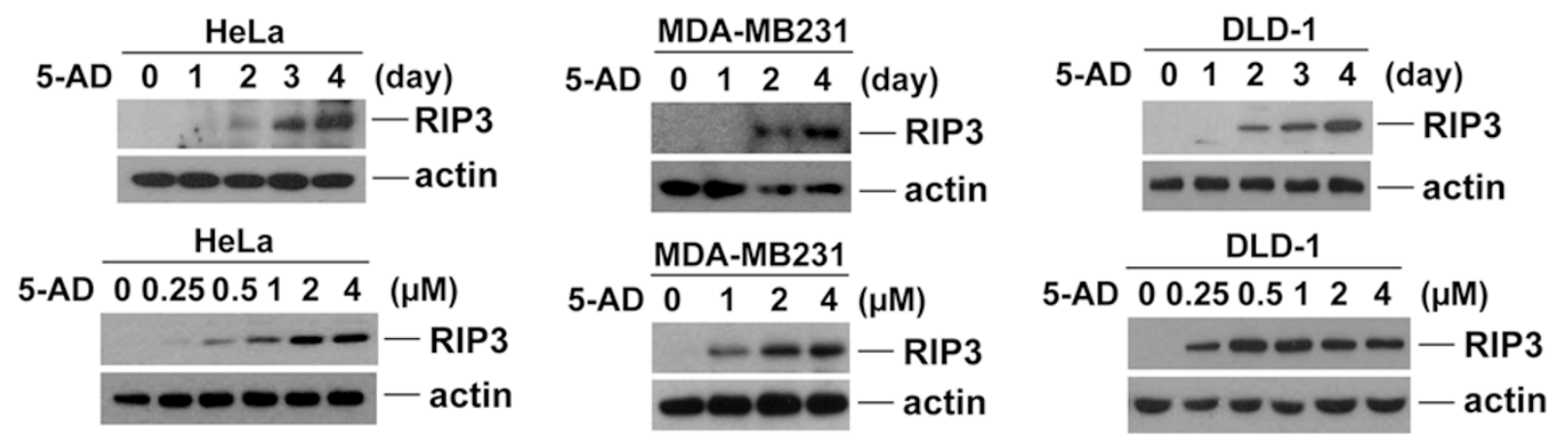

Figure 3 RIP3 is silenced by methylation in multiple cancer cell lines. (A) Western blotting analysis of lysates from multiple cancer cell lines showing RIP3 expression. (B) Western blotting analysis of RIP3 expression is shown in melanocyte, keratinocyte and fibroblasts from human normal skin. Other lysates from human mammary epithelial cells (HMLE: immortalized, hTERT + T antigens; HME: primary), primary prostate epithelial cells (two specimens: PPE7, PPE30), normal hepatocyte cell line HL7702 and primary brain tissue (two specimens: E3, E37) were also probed for RIP3 expression. (C) HeLa, A549, and HT-29 cells were either lysed in M2 buffer (left) or directly dissolved and boiled in Laemmli SDS buffer (right) and lysates were analyzed by western blot. Blotting for Vimentin is included as a positive control for detection of insoluble protein. (D) Immunoblotting showing NIK protein expression in HeLa and DLD-1 cells upon proteosome inhibition with MG132 $(10 \mu \mathrm{M})$. (E) Western blotting analysis of HeLa cells treated with sodium butyrate $(2.5 \mathrm{mM})$ or Trichostatin $\mathrm{A}(0.25 \mu \mathrm{g} / \mathrm{ml})$ for 1 day in the presence or absence of 5-AD. (F) Western blotting analysis of RIP3 expression in HeLa, MDA-MB231, and DLD-1 cells treated with $5-\mathrm{AD}$ for the indicated times $(2 \mu \mathrm{M})$ and with different concentrations (4 days). 
A

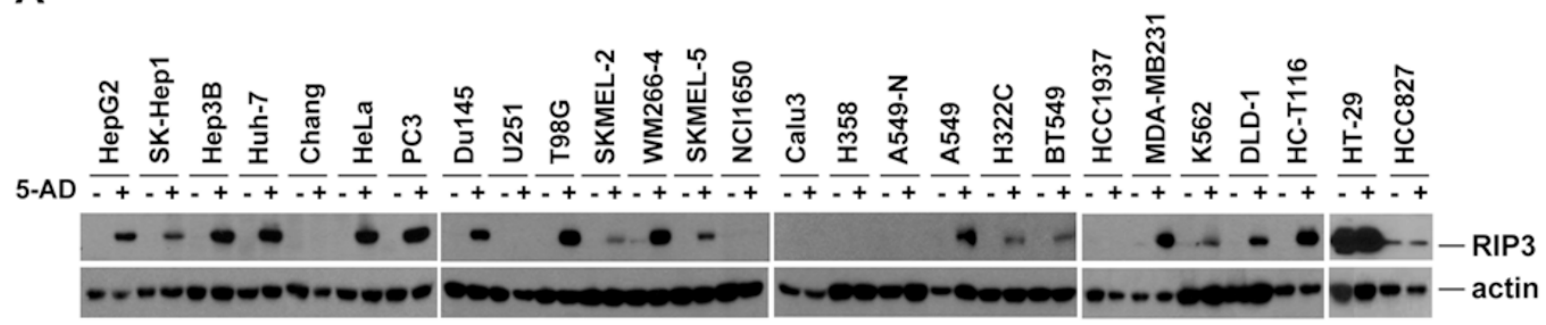

B

HeLa DLD-1 HCT-116

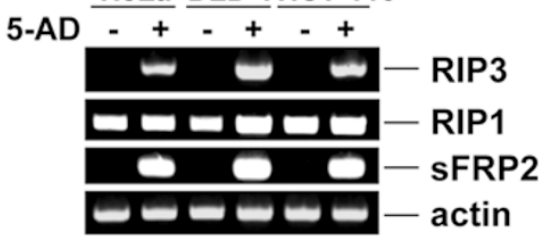

D

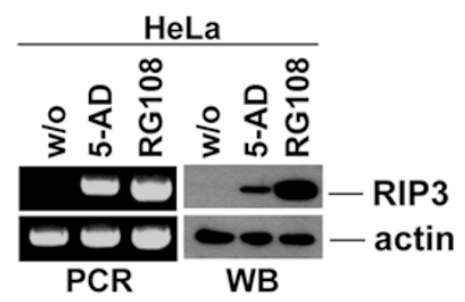

C

HeLa DLD-1 HeLa DLD-1

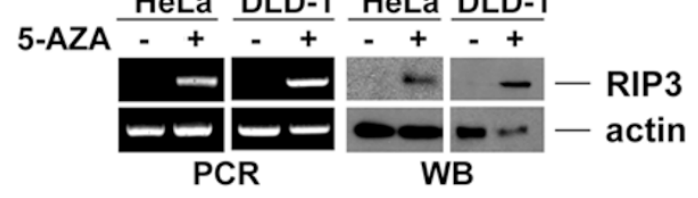

E

MDA-

MB231 K562 HeLa HT-29

$\overline{M U} \overline{M U} \overline{M U} \overline{M U}$

$\square \quad \infty \quad-$ RIP3

F

\begin{tabular}{|c|c|c|c|c|c|}
\hline HeLa & DLD-1 & HCT-116 & MDA-MB231 & HeLa & DLD-1 \\
\hline w/o +5-AD & $w / 0+5-A D$ & w/o +5-AD & w/o +5-AD w/o +5-AD & $w / o+5-A D$ & $w / o+5-A D$ \\
\hline$M \cup M U$ & $M U M U$ & $M U M U$ & $M \cup M U M \cup M U$ & $\overline{M U} \overline{M U}$ & $\overline{M U} \overline{M U}$ \\
\hline 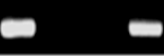 & $c=-4$ & 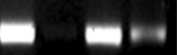 & - & $\infty \quad \pi=$ & $\theta$ \\
\hline
\end{tabular}

G

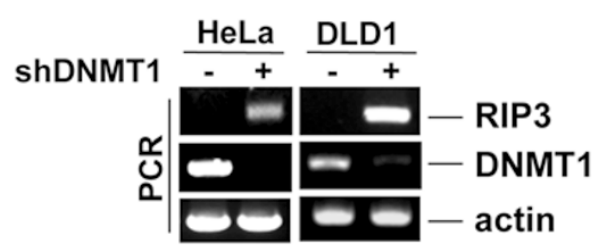

H

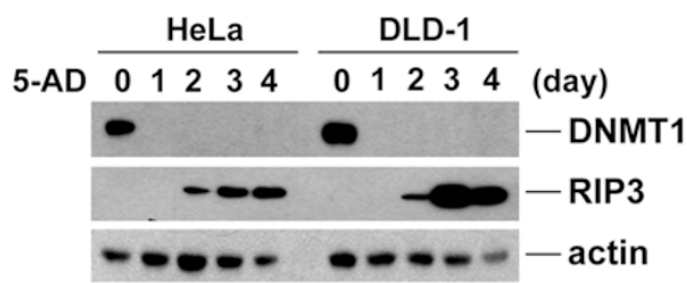

Figure 4 Methylation of the RIP3K TSS is negatively associated with RIP3 expression in cancer cell lines. (A) Western blotting showing the response of various cancer cells to $5-A D(2 \mu \mathrm{M}, 4$ days). (B) Reverse transcription-PCR products from cells following treatment with 5-AD $(2 \mu \mathrm{M}, 4$ days). A sFRP2 product is shown as a positive control for methylation. (C) Western blotting and reverse transcription-PCR results after 5-AZA treatment of HeLa (0.625 $\mu \mathrm{M}, 8$ days) or DLD-1 (1.25 $\mu \mathrm{M}$, 4 days) cells. (D) Western blotting and reverse transcription-PCR results after treatment of HeLa cells with $5-A D(2 \mu M, 4$ days) or RG108 (100 $\mu \mathrm{M}, 8$ days). (E) Methylation-specific PCR (MSP) of genomic DNA from HeLa, MDA-MB231, K562, and HT-29 cells. $M$ and $U$ indicate methylated-specific and unmethylated-specific primers, respectively, to the RIPK3 sequence. (F) MSP of genomic DNA from HeLa, DLD-1, HCT-116, MDA-MB231, and K562 cells treated with 5-AD (2 $\mu$ M, 4 days). MSP was repeated on genomic DNA from HeLa and DLD-1 cells with a second set of methylated- and unmethylated-specific primers. (G, H) HeLa and DLD-1 cells were infected with a lentivirus encoding DNMT1 shRNAs, or non-silencing control. After selection with puromycin, cells with stable shRNA expression were analyzed by reverse transcription-PCR (G) or western blotting $(\mathbf{H})$ after 5-AD $(2 \mu \mathrm{M})$ treatment of HeLa or DLD-1 cells, showing reverse correlation between DNMT1 and RIP3 expression. 
knockdown of DNMT3a or DNMT3b did not result in increases in RIP3 mRNA either alone or when used in combination with DNMT1 knockdown (Supplementary information, Figure S7C-S7E). Interestingly, when A549 or A549-N cells were treated with 5-AD, there was no observable loss in DNMT1 mRNA in A549-N cells as in A549 cells, consistent with their lack of response to 5-AD (Supplementary information, Figure S7F). When DNMT1 was overexpressed in $\mathrm{H} 2009$ cells, which have endogenous RIP3 expression, RIP3 expression was significantly reduced (Supplementary information, Figure S7G). The DNA methylation data, the response of RIP3 expression to 5-AD, and the reverse correlation between DNMT1 and RIP3 protein expression, suggest that DNMT1 is the methyltransferase that is responsible for maintaining RIP3 silencing in cancer cell lines.

Methylation of the genomic region near the TSS of RIPK3 regulates RIP3 expression in human AML patients

RIP3 is not frequently silenced in hematopoetic cancer cell lines (Figure 3A), thus RIP3 methylation is not expected to be prevalent in blood-related cancers. Nevertheless, we examined samples from patients with AML, a disease often characterized by aberrant methylation and sometimes treated with hypomethylating agents. RIP3 was expressed significantly at protein levels in the blood of non-cancer patients; however, bone marrow obtained from four of nine AML patient samples had low RIP3 protein and mRNA expression (Supplementary information, Figure S8A and S8B). This largely corresponded to the level of methylation downstream of the RIPK3 TSS in these patients; all patients with a methylated/ unmethylated DNA ratio $>50 \%$ had low expression of RIP3 (Supplementary information, Figure S8B and $\mathrm{S} 8 \mathrm{C})$. When we examined the expression of DNMTs in these patients (Supplementary information, Figure S8D), DNMT1 was somewhat correlated with the RIPK3 methylation status (Supplementary information, Figure $\mathrm{S} 8 \mathrm{E})$. In one AML clinical trial in which we were able to obtain PBMC samples before and after treatment (5AZA and lenolidomide), we found only one patient with low RIP3 mRNA expression. RIP3 mRNA in this patient was significantly increased in the post-treatment sample, indicating response to the hypomethylating agent (Supplementary information, Figure S8F). MethylCap-sequencing in a separate group of $10 \mathrm{AML}$ patients indicated that the detectable RIPK3 methylation of four patients was eliminated in response to 5-AD treatment, while one patient gained methylation after treatment (Supplementary information, Figure S8G). Thus, despite that AML is likely a suboptimal cancer type to examine RIP3 silencing, the AML data provide proof of principle that RIPK3 methylation controls RIP3 expression in human patients, and that hypomethylating agents could potentially be used to manipulate RIPK3 methylation and expression in vivo.

\section{RIP3 expression is lost in primary breast cancers}

We next examined RIP3 expression in primary breast cancer samples by immunoblotting. Approximately $80 \%$ of the patients had significantly less expression of RIP3 protein in tumor samples than normal tissue (Figure 5A). Immunohistochemical (IHC) analysis of RIP3 gave similar results to western blotting (Figure 5B), and mRNA expression was correlated with protein expression (Supplementary information, Figure S9A). In IHC data from 75 breast cancer patients, RIP3 was reduced in tumor compared to normal tissue, with many tumors lacking detectable RIP3 (see Supplementary information, Figure S9B for scoring comparisons between paired samples; Figure 5B, right panel, for average histological scoring). All cancer subtypes had decreased expression of RIP3, with luminal A (73\%) and luminal B (84\%) subtypes slightly less likely to be RIP3 deficient than triple negative $(90 \%)$ and Her2-positive (95\%) cancers (Figure 5C), and as a group, ER-positive tumors were slightly less likely to be RIP3 deficient than ER-negative tumors (Supplementary information, Figure S9C). Methylation of the genomic region near the RIPK3 TSS was increased in tumor compared to normal samples, showing that RIP3 deficiency was correlated with methylation (Figure 5D). We would expect that the few patients with tumor RIP3 expression could respond better to chemotherapy than their counterparts, and therefore have better outcomes. Unfortunately, many gene expression arrays previously used in breast cancer outcome studies lacked RIP3 probe sets. However, using the bc-GenExMiner tool designed by Jézéquel et al. [36], we analyzed combined data available from 6 studies (1 166 patients). Patients with greater than median RIP3 mRNA expression had a higher probability $(P<0.0085)$ of metastatic relapse-free survival over a 10-year period (Figure 5E). Unfortunately, this tool groups the patients only into two groups above and below the median (50\% split), rather than in quartiles or other smaller divisions. Because RIP3 expression is very low in $>85 \%$ of tumors (Supplementary information, Figure S9B), this means that $\sim 30 \%$ of patients that have low RIP3 expression are wrongly classified into the upper $50 \%$ curve as RIP3 proficient, thus the upper curve of the Kaplan-Meier plot contains many patients that are RIP3 deficient, and the plot underestimates the prognosis for the breast cancer patients that have normal RIPK3 expression. 
A

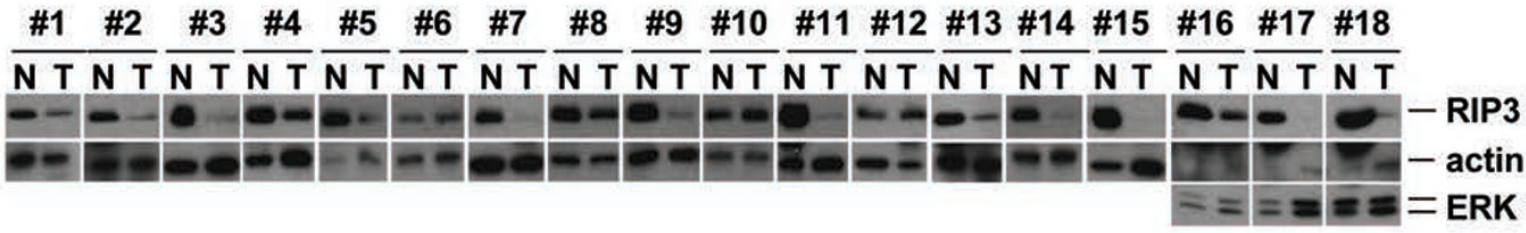

B

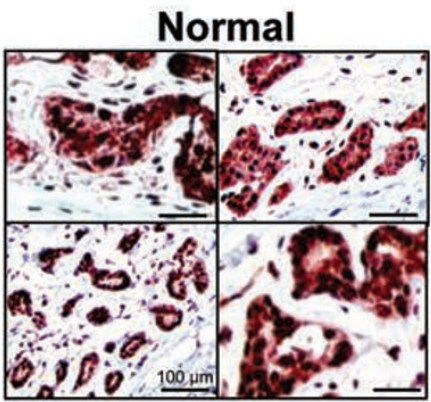

Tumor
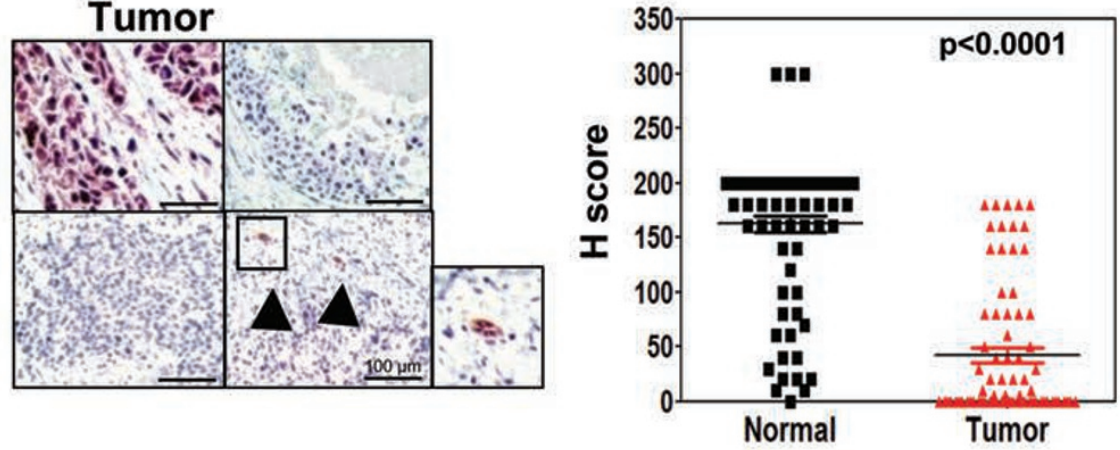

C
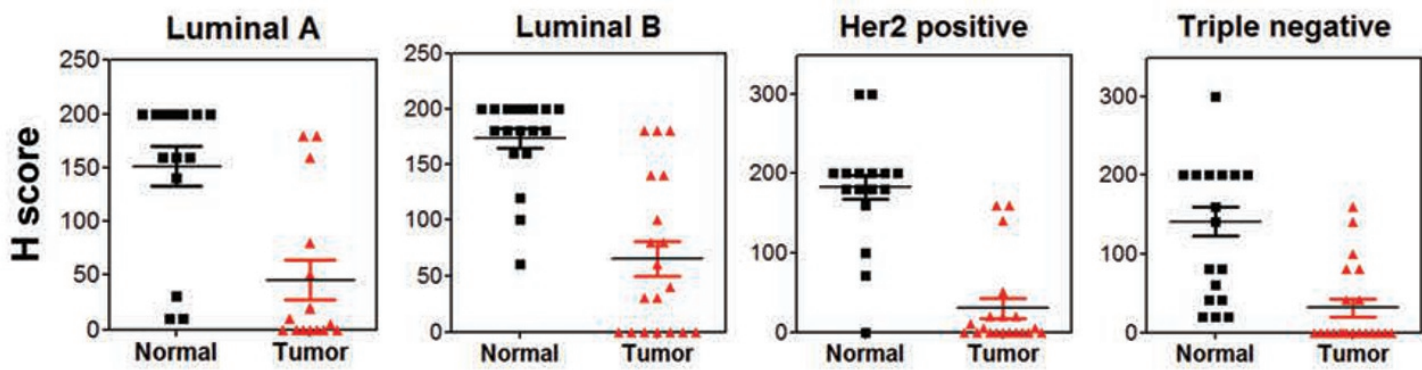

\begin{tabular}{|c|c|c|c|c|}
\hline H score & Normal>Tumor & Normal=Tumor & Normal<Tumor & $\begin{array}{c}\text { Total } \\
(n=75)\end{array}$ \\
\hline Luminal A & $73 \%(n=11)$ & $13.3 \%(n=2)$ & $13.3 \%(n=2)$ & 15 \\
\hline Luminal B & $84.21 \%(n=16)$ & $10.53 \%(n=0)$ & $5.26 \%(n=1)$ & 19 \\
\hline Her2 positive & $95 \%(n=19)$ & $5 \%(n=1)$ & $0 \%(n=0)$ & 20 \\
\hline Triple negative & $90.476 \%(n=19)$ & $9.523 \%(n=2)$ & $0 \%(n=0)$ & 21 \\
\hline
\end{tabular}

D

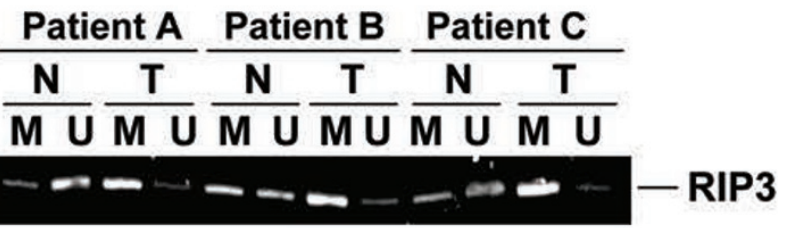

E

RIPK3

Kaplan-Meier survival estimates for pool

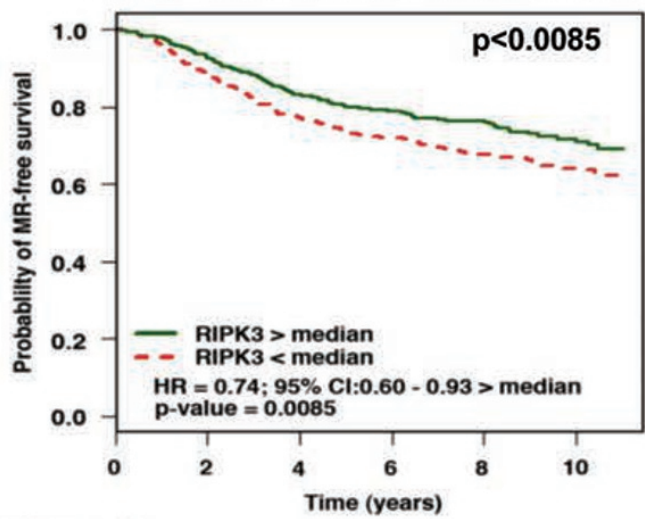

Patients at risk:

$$
\begin{array}{rrrrrr}
582 & 507 & 429 & 305 & 189 & 93 \\
--584 & 486 & 403 & 300 & 203 & 112
\end{array}
$$


Hypomethylating agents restore programmed necrosis in cancer cells through reversal of RIP 3 silencing

The response of cells to 5-AD suggests that RIP3 expression may be manipulated in cancer cells to make them sensitive to programmed necrosis. When treated with 5-AD, multiple cell types previously refractory to TNF- $\alpha$-induced necrosis became sensitive (Supplementary information, Figure S10A-S10C). This was accompanied by sustained activation of JNK (Supplementary information, Figure S10D), which is often associated with programmed necrosis [29, 30], and RIP3 was found in association with RIP1 in the necrosome complex (Supplementary information, Figure S10E). Importantly, 5-AD did not sensitize RIP3-expressing HT-29 and L929 cells to TNF- $\alpha$-induced necrotic cell death (Supplementary information, Figure S11A). Moreover, while 5-AD sensitized A549 cells to TNF-induced necrosis, a variant of A549 (designated A549-N) that upregulates sFRP2 but not RIP3 in response to 5-AD, remained resistant (Supplementary information, Figure S11B). Notably, other hypomethylating agents that restored RIP3 expression similarly sensitized HeLa cells to TNF- $\alpha$-induced necrosis (Supplementary information, Figure S11C). Signaling pathways downstream of death receptor activation were minimally affected by $5-\mathrm{AD}$, since TNF- $\alpha$ - or TRAIL-induced I $\mathrm{B} \alpha \alpha$ degradation and transient JNK phosphorylation remained normal (Supplementary information, Figure S11D). Multiple shRNAs preventing RIP3 upregulation blocked 5-AD-mediated sensitization to TNF- $\alpha$-induced necrosis (Supplementary information, Figure S11E) and RIP3 shRNA inhibited TNF- $\alpha$-induced necrosis in HT29 cells to a similar extent irrespective of whether or not 5 -AD was present (Supplementary information, Figure S11F). These data collectively indicate that hypomethylating agents restore TNF- $\alpha$-induced programmed necrosis in cancer cells solely through reversal of RIP3 silencing and not through additional mechanisms.
Hypomethylating agents sensitize RIP3-deficient cells to chemotherapeutic agents in a RIP3-dependent manner

We next sought to test whether hypomethylating agents would sensitize RIP3-deficient cells to chemotherapeutic agents via restoration of RIP3 expression. 5-AD treatment sensitized multiple RIP3-deficient cell lines both to etoposide and to doxorubicin, as measured by multiple assays (Figure 6A and 6B, Supplementary information, Figure S12A-S12F), and 5-AZA similarly sensitized multiple cell lines to doxorubicin and etoposide (Supplementary information, Figures S12F, S13A and S13B), as well as CPT and actinomycin D (Supplementary information, Figure S13A, right panel). Hypomethylating agents also sensitized RIP3-deficient cell lines to other drugs, including paclitaxel, 5-FU, CPT, and cisplatin (Supplementary information, Figure S13C and S13D; data not shown), similar to the experiments in cells with ectopic RIP3 expression (Figure 1C).

Importantly, 5-AD failed to sensitize RIP3-expressing HT-29 cancer cells and HDF normal cells to the doxorubicin and etoposide, as well as other drugs (Figure 6C and 6D). Likewise, 5-AD failed to chemosensitize $R I P 3^{-/-}$MEFs (Supplementary information, Figure $\mathrm{S} 13 \mathrm{E}$ ) as well as A549-N cells (Figure 6E), which upregulated SFRP2 but not RIP3 in response to 5-AD (Supplementary information, Figures S11B, S6B and S7F). Thus 5-AD appeared to act through induction of RIP3 expression. Crucially, RIP3 shRNA inhibited 5-AD potentiation of doxorubicin- and etoposide-induced cell death in HeLa cells (Figure 6F). Likewise, 5-AD and 5-AZA potentiation of doxorubicin toxicity was inhibited by RIP3 shRNA in K562 cells (Supplementary information, Figure S14A and S14B). In the absence of RIP3, 5-AD also lost its ability to sensitize MDA-MB231 cells to multiple chemotherapeutic agents as measured by different assays (Figure 6G and Supplementary information, Figure S14C), confirming that their sensitization is

Figure 5 RIP3 is silenced in breast cancer. (A) Western blotting for RIP3 expression is shown for breast cancer tumors compared to normal tissue from the same patients ( $N$, normal tissue; $T$, tumor tissue). In 3 cases where the actin immunoblots had problems, ERK protein is shown as a protein loading control. (B, C) Immunohistochemical staining of RIP3 in paraffinembedded tissue specimens from tumor and non-tumor samples in breast cancer patients. Arrows indicate entrapped normal ducts. Paired tumor and normal tissue from each patient was quantitated (B, right). Intensity of histological staining grade was quantitated according to American Society of Clinical Oncology (ASCO)/College of American Pathologists (CAP) guidelines using the following categories: 0 , no immunostaining; $1+$, weak incomplete membranous staining, $<10 \%$ of tumor cells; $2+$, complete membranous staining, either uniform or weak in at least $10 \%$ of tumor cells; and $3+$, uniform intense membranous staining in at least $30 \%$ of tumor cells. Staining grade is shown for various subtypes of breast cancer (C). Scale bar, 100 $\mu \mathrm{m}$. (D) Methylation-specific PCR of genomic DNA from tumor and non-tumor tissue of three triple-negative breast cancer patients (patients A-C). M and $U$ indicate methylated-specific and unmethylated-specific primers (set 1), respectively, to the RIPK3 genomic sequence. (E) Kaplan-Meier curve showing metastasis relapse (MR)-free survival of breast cancer patients having above or below median expression of RIP3 (RIPK3). Hazard ratio (HR) based on relative risk of event occurrence between the group of patients with RIP3 expression above the median value in the selected population and the group with RIP3 expression below the median value. 
A

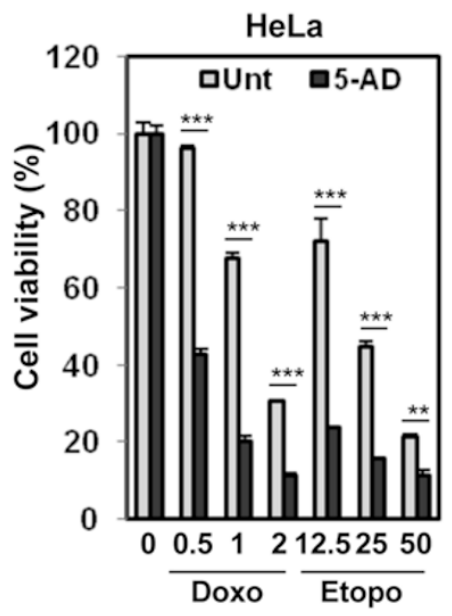

C

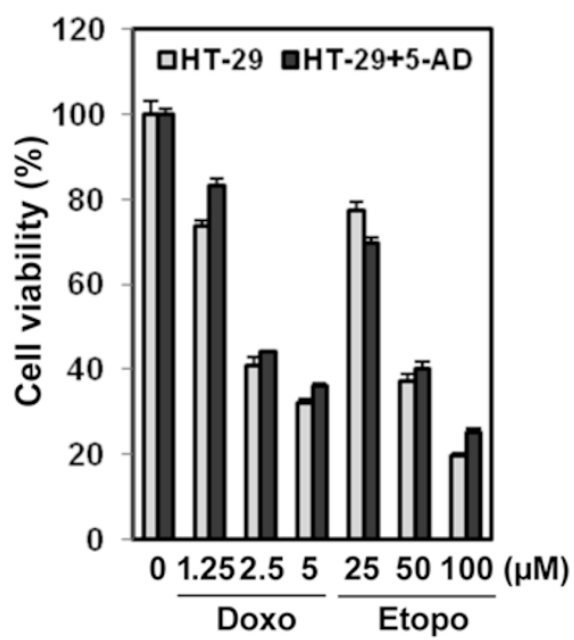

$\mathbf{F}$

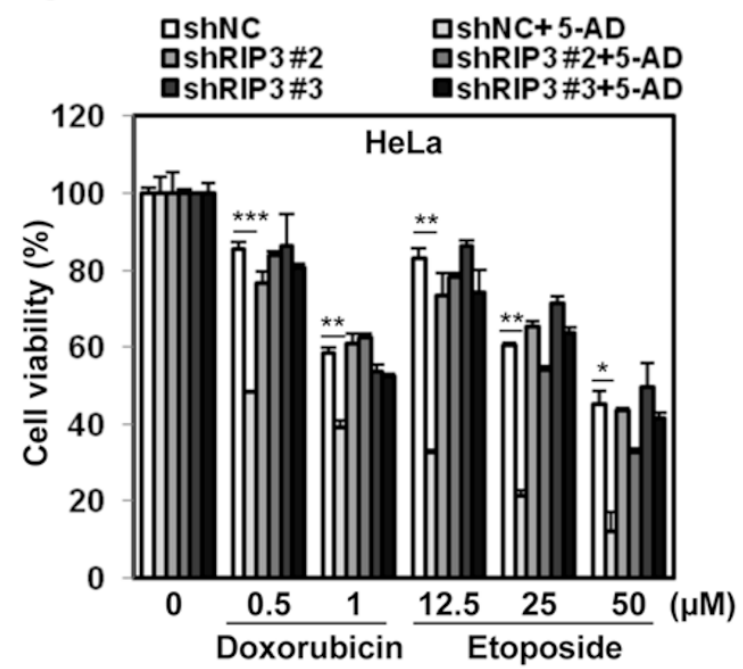

B

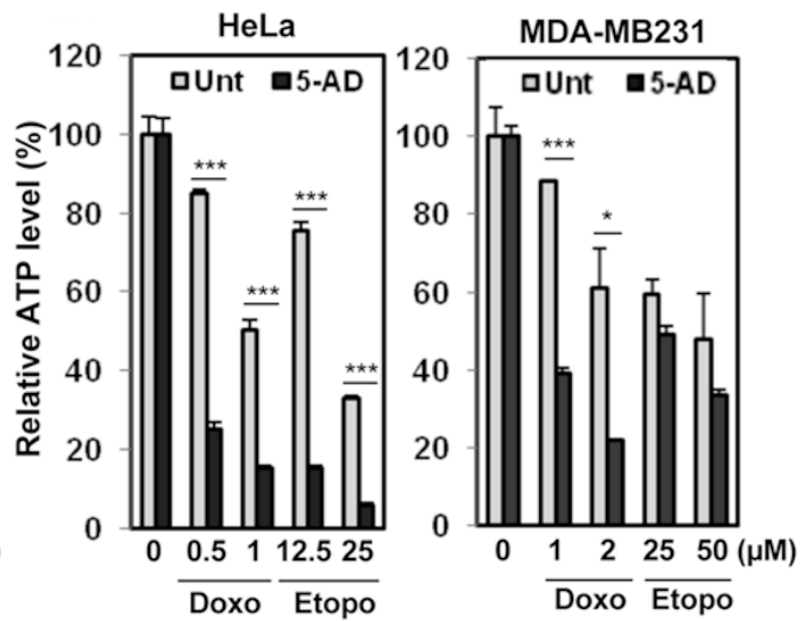

E

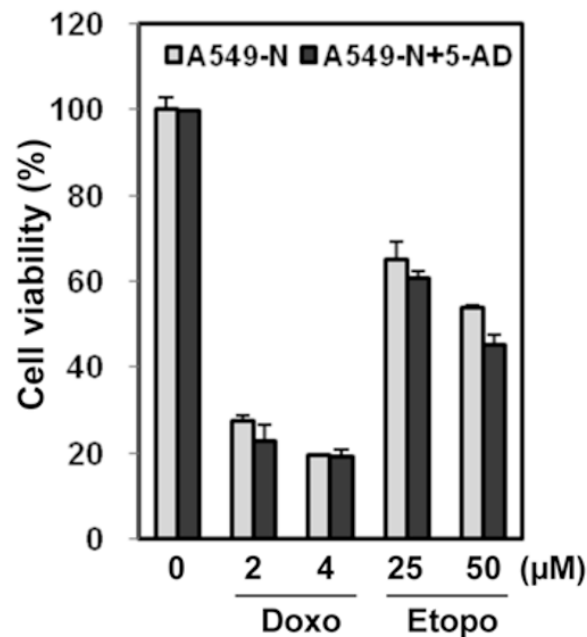

G

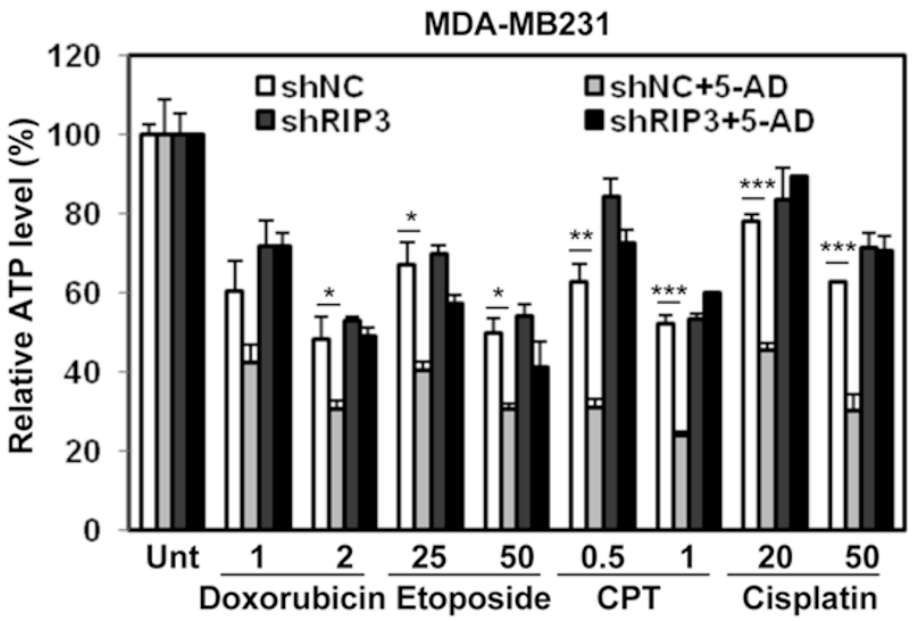


dependent on the induction of RIP3 expression. Taken together, these data suggest that hypomethylating agents promote chemosensitivity in these cell types largely through the induction of RIP3 expression.

\section{5-AD synergizes with doxorubicin in breast tumor treatment in vivo}

Lastly, an orthotopic MDA-MB231 xenograft tumor model was used to examine whether 5-AD would promote chemosensitivity in vivo. Mice were treated with 5-AD, doxorubicin, or both, with doxorubicin and 5-AD doses such that they had minimal effect by themselves on tumor growth (Figure 7A and 7B). The combination of 5-AD and doxorubicin resulted in a synergistic reduction in tumor size and tumor mass (Figure 7A and 7B), with no appreciable toxicity in the liver (Figure 7C). TUNEL staining and IHC staining of p-H2AX indicated a significantly greater cytotoxicity in the tumors of the doxorubicin/5-AD-treated mice than in the tumors of mice treated with a single drug (Figure 7D). RIP3 mRNA was highly expressed in the tumors of most of the 5-AD-treated mice, irrespective of whether or not mice were treated with doxorubicin (Figure 7E) and RIP3 protein was clearly detected in tumors of doxorubicin/5AD-treated mice, but not untreated mice (Figure 7F). These data suggest that 5-AD was effective in restoring RIP3 expression in tumor cells in vivo, and that it can synergize with doxorubicin to reduce the tumor burden of RIP3-deficient breast cancer in vivo.

\section{Discussion}

Our data indicates that RIP3 contributes to the cytotoxicity of chemotherapeutic agents with disparate mechanisms of action, including topoisomerase inhibitors, taxanes, platinum complexes, anthracyclines, and antimetabolites through induction of programmed necrosis. How RIP3 is activated by drugs with such mechanistic differences is an important matter for future investigation. A role for RIP1 in genotoxic-induced cell death has been suggested in several previous publications $[26,37]$. A RIP1-dependent complex was previously reported to play a role in etoposide-induced cell death through the activation of both apoptotosis and necrosis, however, in that report RIP3 was described as contributing to caspase activation [26] through the "ripoptosome", a very large complex containing RIP1, FADD, and caspase-8, as well as caspase- 10 and cFLIP $[26,38]$ that is assembled after treatment with IAP antagonists [38]. The ripoptosome is similar to the secondary complex (TNF-R1 complex II) formed downstream of TNF-R1 activation [39], but its formation does not require TNF-R1. Our data also suggests the formation of a similar complex in response to chemotherapeutics, and we also did not observe TNF-R1 involvement. Interestingly, TRADD was recruited to the complex, which suggests additional similarity to TNF-R1 complex II, though these complexes may originate by different mechanisms. Since a previous report indicated that depletion of cIAP proteins was key to the formation of the ripoptosome, cIAP depletion may indicate a common mechanism for formation of this complex induced by chemotherapeutic drugs with different mechanisms of action [38]. We have not yet examined whether this is a common occurrence with all stimuli. However, if so, it remains uncertain how cIAP depletion would occur after treatment of all of these different drugs. JNK activation contributes to a common mechanism after treatment of all of these different agents, since JNK activity is known to accompany, and sometimes be required for, the programmed necrotic pathway in many instances [29, 30]. However, we found that JNK activity was highly enhanced in RIP3-expressing cells in response to various chemotherapeutics, suggesting that JNK activation is downstream of the necrosome complex formation.

Unlike the previous papers mentioned above, we were able to immunoprecipitate RIP3 with the RIP1 complex.

Figure 6 Restoration of RIP3 by hypomethylating agents enhances sensitivity to DNA-damaging agents. (A, B) HeLa and MDA-MB231 cells were treated with 5-AD (2 $\mu \mathrm{M}, 4$ days), then with doxorubicin or etoposide for $48 \mathrm{~h}$ and cell viability was analyzed by MTT assay (A) or ATP depletion (B). Results are presented as means \pm SEM. (C-E) HT-29 cells, which endogenously express RIP3, were treated with 5-AD ( $2 \mu \mathrm{M}, 4$ days) and then with doxorubicin or etoposide for $48 \mathrm{~h}$, and cell viability was analyzed by MTT assay (C). Human dermal fibroblasts (HDF), which are untransformed and have RIP3 expression, were treated with $5-\mathrm{AD}(2 \mu \mathrm{M}, 4$ days), then with doxorubicin $(2 \mu \mathrm{M})$, etoposide $(25 \mu \mathrm{M}), 5-\mathrm{FU}(50 \mu \mathrm{M})$, or CPT $(1 \mu \mathrm{g} / \mathrm{ml})$ for $48 \mathrm{~h}$ and cell viability was analyzed by MTT assay (D). A549-N cells, which do not upregulate RIP3 expression in response to hypomethylating agents, were treated with 5-AD $(2 \mu \mathrm{M})$ for 4 days and cell viability was analyzed by MTT assay (E). Results are presented as means \pm SEM. (F) HeLa cells with stable shRNA expression were pretreated with $5-A D(2 \mu M$, 4 days) and then treated with doxorubicin or etoposide for 2 days and cell viability was analyzed by MTT assay. Results are presented as means \pm SEM. (G) MDA-MB231 cells with stable shRNA expression were pretreated with 5-AD (2 $\mu$ M) for 4 days and then treated with doxorubicin $(\mu \mathrm{M})$, etoposide $(\mu \mathrm{M}), \mathrm{CPT}(\mu \mathrm{g} / \mathrm{ml})$, and cisplatin $(\mu \mathrm{M})$ for $48 \mathrm{~h}$ and cell viability was analyzed by relative ATP level analysis. Results are presented as means \pm SEM. ${ }^{*} P<0.05,{ }^{* *} P<0.01,{ }^{* * *} P<0.001$. 


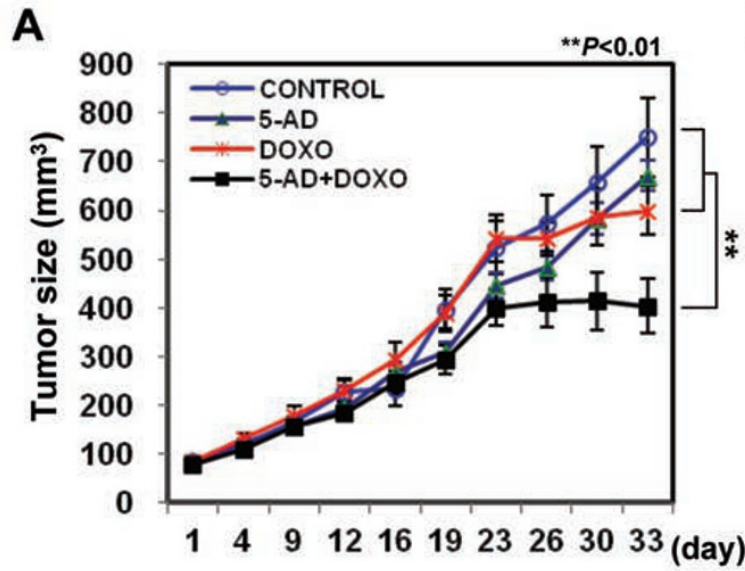

B
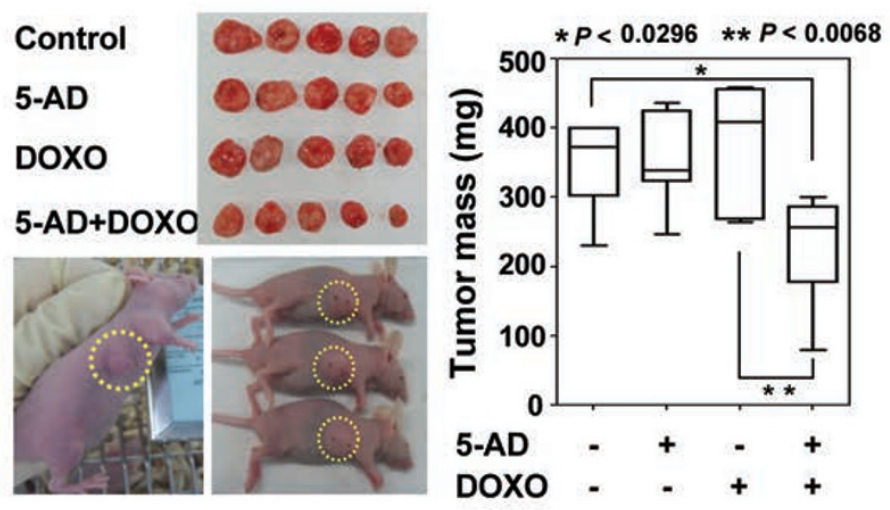

C

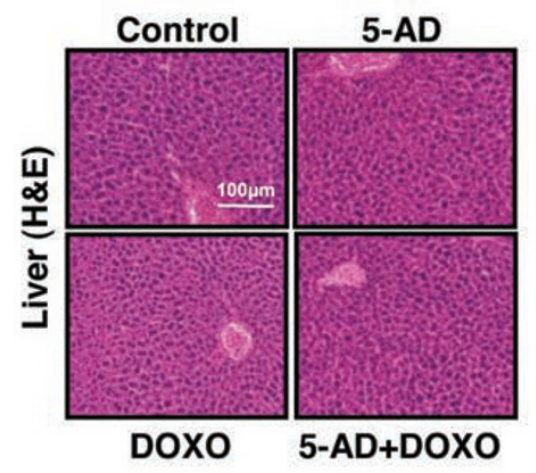

D

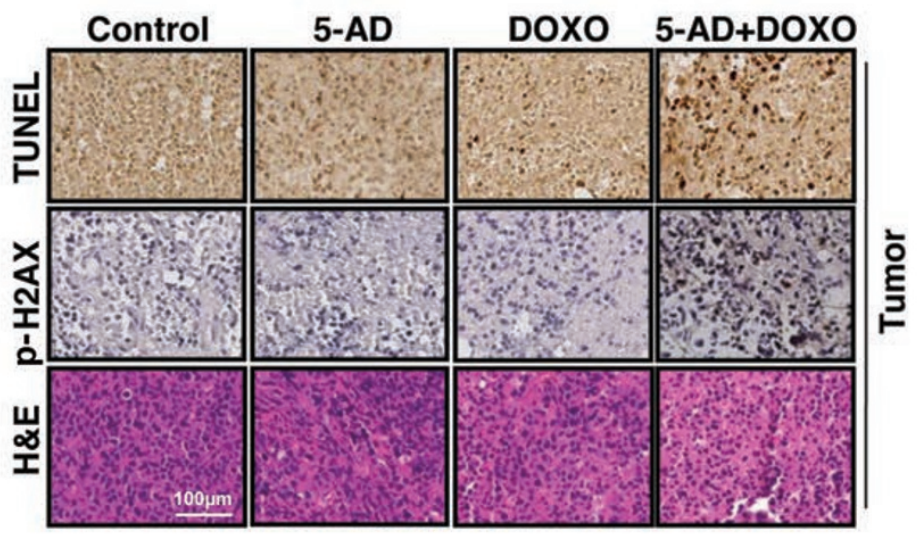

$\mathbf{F}$
E

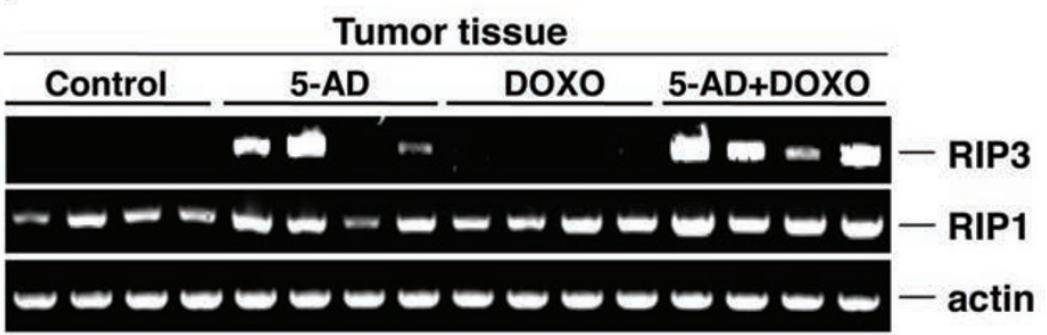

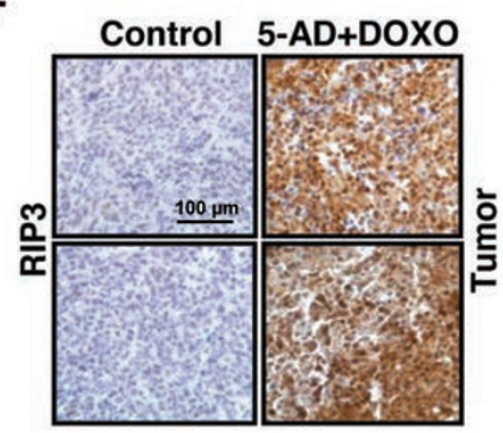

Figure 7 The combination of 5-AD plus doxorubicin inhibits breast cancer growth in a mouse xenograft model. (A) Effect of 5-AD plus doxorubicin on MDA-MB231 breast cancer cell xenograft in athymic BALB/C nude mice. MDA-MB231 tumors were established orthotopically and treated with 5-AD for the first week and then doxorubicin for 33 days. Tumor volume was monitored and is shown in the graph. Data are presented as means $\pm \mathrm{SD}(n=7)$. (B) At the end of treatment mice were killed and tumors are shown (left). Tumor mass was measured and is summarized (right). (C) H\&E staining for liver section of mice receiving the indicated treatment. (D) Top panel shows detection of cell death by TUNEL assay in tumor sections of mice receiving the indicated treatment. Sections were also stained for p-H2AX and H\&E. (E) PCR of tumor tissues to detect RIP3 restoration upon 5-AD injection in mice. (F) Immunohistochemical staining of RIP3 in paraffin-embedded tissue specimens from tumor of mouse xenograft.

We found that complex formation led to MLKL activation and programmed necrosis rather than caspase- 8 activation and apoptosis. Interestingly, though caspases are well known to promote programmed necrotic cell 
death mediated by death receptors [4], zVAD did not potentiate the genotoxic compound-induced necrosis in our experiments. This may be because in the case of chemotherapeutics, the caspase activation does not emanate solely from one complex, so does death receptor-mediated caspase- 8 activation. Since genotoxic stress is known to trigger caspase- 9 and caspase-3 activation downstream of mitochondrial permeabilization, zVAD may prevent the part of the cell death that is caspase dependent, leading to both potentiation and inhibition of cell death by zVAD.

A role for programmed necrosis in cell death resulting from DNA damage by alkylating agents was first suggested over a decade ago; however, in that context, the DNA repair protein poly(ADP-ribose) polymerase (PARP) was required for cell death [40]. Recent studies have suggested that multiple distinct mechanisms exist for programmed necrosis to occur [13, 41], and that PARP-dependent necrotic cell death, which has been referred to as "parthanatos", and the RIP1-RIP3-dependent process induced by TNF, which has been referred to as "necroptosis", are largely separate pathways [41], though they both lead to programmed necrotic outcomes. Although necrostatin-1, necrosulfonamide or dabrafenib, unlike zVAD, provides protection from etoposide- and doxorubicin-induced cell death in our assays, this protection is not complete, suggesting that RIP3-independent mechanisms of programmed necrotic death could also be involved.

Tumor formation often selects against the expression of cell death proteins, and resistance to cell death is one of the hallmarks of a cancer cell [1]. One way by which expression of such a protein can be regulated is through methylation of its genomic DNA, thus silencing its transcription. This appears to be the case with RIP3. Since RIP3 expression is lost in most cancer cell lines and is low in about $85 \%$ of primary breast cancer samples that we have examined, but RIP3 is present in normal tissue and in primary cells (Figure 4B, [42-44]), this would suggest that RIP3 expression is negatively selected during initial tumor development or tumor growth. Loss of RIP3 in cancer cells (or in highly passaged cell lines, such as non-tumorigenic MCF10A and MCF12A) is understandable since such cells are under strong selective pressure, and given the essential role of RIP3 in promoting programmed necrotic cell death, loss of RIP3 may provide a selective advantage during growth.

The analysis of RIPK3 methylation from our data and the publicly available databases (such as the University of California-Santa Cruz Genome Browser, among others), combined with our RIP3 protein expression data, suggests that the correlation between RIP3 silencing and the methylation of a given $\mathrm{CpG}$ is strongest very near the TSS, and is much reduced a few hundred basepairs downstream of the TSS (mostly correlated with the methylation status of four proximal CpGs just downstream of the RIP3K TSS). Although we cannot exclude the presence of enhancer sequences within this region of the genome, the correlation of methylation near the TSS may suggest that methylation may directly interfere with the DNA binding of the main transcriptional machinery.

Importantly, RIP3 expression may be restored in most cells by use of simple hypomethylating agents, such as 5-AD, enabling the manipulation of RIP3 expression in both cell lines and patients, and thus sensitization to programmed necrotic cell death. Multiple clinical trials of 5-AZA as a single agent therapy have previously demonstrated a response plus stable disease rate of about $35 \%$ in breast cancer patients, with a response rate of $18 \%$ [45]. While this response rate may seem small, the response rate for myelodysplastic syndrome (5-AZA has recently been FDA approved for its treatment) is only slightly higher at $23 \%$ [45]. Based on our data, we would predict that the use of hypomethylating agents, such as 5-AZA, would be much more effective when combined with other chemotherapeutic drugs. Since RIP3 sensitizes some cell types to doxorubicin and paclitaxel, both of which are currently used in the clinic for the treatment of breast cancer, we may expect hypomethylating agents to make these drugs more effective in the treatment of RIP3-deficient breast cancers.

Except a few cell lines utilizing ectopic expression of RIP3, the (mostly TNF- $\alpha$-induced) study of programmed cell death has primarily been confined to a few cancer cell line models (e.g., the mouse fibroblast L929 cells (in which TNF-induced necrosis was first observed), a handful of cells from the hematopoietic system, MEFs, and a few epithelial cell lines of the gastrointestinal tract (such as HT-29), leaving researchers to wonder whether these particular cell lines were exceptional, or whether the mechanistic data obtained in these few cell lines is generally applicable. It appears from our data that a majority of cell lines may be sensitized to programmed necrosis by upregulating RIP3 expression with hypomethylating agents, suggesting a conservation of mechanism, and enabling further mechanistic study of programmed necrotic cell death in a wide variety of cell types.

In summary, our data indicate that RIP3 expression is likely negatively selected during cancer development or progression, with methylation of the genomic region near the RIPK3 TSS leading to RIP3 silencing. Since RIP3 plays a greater role in response to chemotherapeutics than previously appreciated, we propose that hypomethylating agents, in combination with standard chemother- 
apy, may be useful in treating cancers that lack RIP3 expression.

\section{Materials and Methods}

\section{Reagents}

TNF- $\alpha$ and zVAD were purchased from R\&D Systems. Antibodies were purchased from the following sources: RIP3 and p-MLKL, Abcam; RIP1 and FADD, BD-Transduction Laboratories; DNMT1 and p53, Santa Cruz Biotechnology; Vimentin, histone-H3, NIK, and caspase-8, Cell Signaling; acetyl-histone-H3 and histone-H4, Millipore; p-JNK, Invitrogen; complex-II, Molecular Probes; MLKL, Sigma-Aldrich. Doxorubicin, etoposide, paclitaxel, 5-FU, cisplatin, CPT, 5-AD, 5-AZA, and necrostatin-1 were all purchased from Sigma-Aldrich (St Louis, MO, USA). MG132, RG108, necrosulfonamide, and cycloheximide were purchased from Calbiochem. Dabrafenib was purchased from Selleckchem. SMAC mimetic (LCL-161) was purchased from Adooq Bioscience.

\section{Cell culture}

Cell lines were cultured as recommended by ATCC. WT and $R_{I P 3^{-/}}$MEF cells and their culture conditions have been previously described [15]. Murine stable cell lines (MEFs and mouse melanoma cells B16) were established by infection with $\mathrm{pBOB}-$ Flag-mRIP3 lentivirus. HeLa cells expressing RIP3 were obtained from cells transfected with Flag-hRIP3 or GFP-hRIP3. RIP3expressing HeLa, MDA-MB231, and Huh-7 cells were from cells infected with pLX303-hRIP3 lentivirus.

\section{Human breast tumor tissue preparation}

Human breast tumor and paired normal samples were obtained from Yonsei University College of Medicine (Seoul, Korea). This study was approved by the Institutional Review Board (IRB) at Yonsei University, and in all cases, informed written consent was obtained from all participants.

\section{Normal human cells}

Normal primary prostate (PPE) and mammary epithelial cells (HME) were originally obtained from Clonetics (San Diego, CA, USA). HMLE are normal mammary epithelial cells that have been immortalized with hTERT and also retrovirally-infected with SV40 large and small T antigens. Normal human melanocytes, keratinocytes and skin fibroblasts were obtained from Gibco/Life Technologies (Grand Island, NY, USA). Primary patient brain samples were obtained from Children's Hospital Colorado and collected in accordance with local and federal human research protection guidelines and institutional review board regulations (COMIRB 95-500). Informed consent was obtained for all specimens collected.

\section{AML patient samples}

AML patient samples obtained from the University of Colorado were obtained with the informed written consent from all participating subjects (COMIRB 06-0720). Bone marrow aspirate was collected in heparin or EDTA tubes. The samples were diluted $4 \times$ with PBS before the addition of Ficoll-Hypaque (SigmaAldrich) density gradient. After centrifugation, the plasma and mononuclear layers were collected and stored at $-80^{\circ} \mathrm{C}$.

\section{Lentiviral shRNA experiments}

MISSION short-hairpin RNA plasmids targeting hRIP3 mRNA (NM_006871), mRIP3 mRNA (NM_019955), hDNMT1 (NM 001379), and hMLKL mRNA (NM 152649), hTNF-R1 mRNA (NM_001065.3) and non-targeting control (NM_027088) were from Sigma-Aldrich. Lentiviral plasmids were transfected into 293TN cells (System Biosciences, LV900A-1) using Lipofectamine 2000 (Invitrogen). Pseudoviral particles were collected 2 days after lentiviral plasmid transfection, and infected cells in the presence of polybrene $(8 \mu \mathrm{g} / \mathrm{ml})$. Cells were selected with puromycin 2 days after infection, and RIP3 knockdown was confirmed by immunoblotting. Cells without endogenous RIP3 were afterwards treated with 5-AD for 4 days and analyzed by immunoblotting.

\section{Western blot and co-immunoprecipitation}

Cell lysis was carried out in M2 buffer [29]. Equal amounts of cell extracts were resolved by SDS-PAGE and analyzed by immunoblotting. For immunoprecipitation, lysates were mixed and precipitated with antibody and protein A-sepharose or protein G-agarose beads overnight at $4{ }^{\circ} \mathrm{C}$. Bound proteins were removed by boiling in SDS buffer and resolved by SDS-PAGE for immunoblotting. Human breast cancer tissues and normal tissues were lysed in M2 buffer, resolved by SDS-PAGE, and analyzed by immunoblotting and blots were visualized by enhanced chemiluminescence (ECL, Amersham).

\section{Confocal microscopy}

Cells were seeded in a coverglass slide chamber after the designated treatments, fixed for $10 \mathrm{~min}$ in $4 \%$ paraformaldehyde, and then stained with p-MLKL antibody (1:250). Cells were mounted with $10 \mathrm{mg} / \mathrm{ml}$ DAPI in aqueous mountant (Dako, Denmark) and viewed by confocal microscopy.

\section{Cytotoxicity assays}

Cell viability was determined using tetrazolium dye colorimetric test (MTT and MTS (Promega) assays with absorbance read at $570 \mathrm{~nm}$ and $490 \mathrm{~nm}$, respectively). Cell survival assays were performed using the CellTiter-Glo Luminescent Cell Viability Assay kit (Promega, G7570) according to the manufacturer's instructions. Luminescence was measured using a POLARstar OPTIMA. For CellTiter-Glo assays, the mean \pm STDEV of duplicates is presented.

\section{Real-time PCR}

Quantitative RT-PCR was performed in Cycler (Applied Biosystems, Foster City, CA, USA) with QuantiTect SYBR Green PCR Kit (Qiagen, Valencia, CA, USA), essentially as described in the manufacturer's instructions, using specific set of primers for RIP3 (PPH15378B, Qiagen). Expression was normalized to SDHA expression (PPH20936F, Qiagen). The relative value of specific mRNAs in each sample was expressed as a reciprocal ratio of the $\mathrm{Ct}$ values of each message to those of the corresponding SDHA.

\section{Reverse transcription- $P C R$}

RNA was extracted using RNeasy (Qiagen). $200 \mathrm{ng}$ of total RNA from each sample was used for cDNA synthesis with reverse transcriptase (Invitrogen). Equal amounts of cDNA product were used for PCR with Taq DNA polymerase (Takara). Amplification was performed using the following primers:

RIP3 sense (5'-CAAGGAGGGACAGAAATGGA-3'), 
RIP3 antisense (5'-GCCTTCTTGCGAACCTACTG-3'); RIP1 sense (5'-CACAGAACCCAGGGACTCAT-3'), RIP1 antisense (5-GACTTCTCTGTGGGCTTTGC-3'); sFRP2 sense (5'-ACGAGACCATGAAGGAGGTG-3'), sFRP2 antisense (5-GATGCAAAGGTCGTTGTCCT-3'); $\beta$-actin sense (5'-CAGGTCATCACCATTGGCAATGAGC-3'), $\beta$-actin antisense (5'-GATGTCCACGTCACACTTCATGA-3'); DNMT1 sense (5'-GTGAGGACATGCAGCTTTCA-3'), DNMT1 antisense (5'-TGCTGCCTTTGATGTAGTCG-3'); TNFR1-forward (5'-TATTG ACTGGTCCCTCACC-3'), TNFR1-reverse (5'-CTGCAATTGAAGCACTGGAA-3'). Final PCR products were resolved in $1.5 \%$ agarose gel and stained with ethidium bromide.

\section{Methylation-specific PCR}

Genomic DNA was extracted using DNeasy Blood \& Tissue kits (Qiagen). Methylation status of RIPK3 was determined by methylation-specific PCR. Briefly, $2 \mu \mathrm{g}$ of genomic DNA was bisulphite-treated with Zymo DNA Modification Kit (Zymo Research, Orange County, CA, USA). Bisulphite-treated DNA was amplified using primers specific for either methylated or unmethylated DNA. The sequences of the first set of methylated-specific (M) primer and unmethylated-specific (U) primer for RIP3 were: RIP3 (M), forward 5'-TAATTCGGAAAAAGGGTAATAATTC-3', reverse 5'-ATAAATATCGAAAACTACGATCGAC-3'; RIP3 (U), forward 5'-AATTTGGAAAAAGGGTAATAATTTG-3', reverse 5'-ATAAATATCAAAAACTACAATCAAC-3'.

The sequences of the second set of methylated-specific (M) primer and unmethylated-specific (U) primer for RIP3 were: RIP3 (M), forward 5'-AGGTTATGGTAAGTTTGGATAACGA-3', reverse 5'-CAACAAACGACAAAAAAACGAC-3'; RIP3 (U), forward 5'-AGGTTATGGTAAGTTTGGATAATGA-3', reverse 5'-CTTTCAACAAACAACAAAAAAACAA-3'.

\section{Pyrosequencing of bisulfate-converted genomic DNA}

Pyrosequencing of RIPK3 Genomic DNA from cells with and without 5-AD treatment was performed by Genomictree (Daejeon, South Korea) using standard protocols. Briefly, bisulfite-treated DNA samples were prepared using EZDNA Methylation-Lightning kit (Zymo Research) and then amplified by PCR. The reaction conditions were as follows: $95^{\circ} \mathrm{C}$ for $10 \mathrm{~min} \rightarrow\left(95^{\circ} \mathrm{C}\right.$ for $30 \mathrm{~s} \rightarrow 56{ }^{\circ} \mathrm{C}$ for $30 \mathrm{~s} \rightarrow 72{ }^{\circ} \mathrm{C}$ for $\left.30 \mathrm{~s}\right) 45$ cycles $\rightarrow 72{ }^{\circ} \mathrm{C}$ for 5 min. From this reaction, $2 \mu$ of the PCR product was visualized by ethidium bromide staining on a $2 \%$ agarose gel. The remainder of the product underwent pyrosequencing performed on a PyroMark ID system (Qiagen) using a PyroGold reagent kit for quantification of the methylation level.

\section{Immunohistochemistry and TUNEL staining}

Immunohistochemistry was performed using the UltraVision LP Detection System TL-060-HD (Thermo Scientific, Bioanalytica) according to the manufacturer's instructions. Thin paraffin sections $(4.5 \mu \mathrm{m})$ were deparaffinized in xylene and rehydrated in a graded series of ethanol-aqueous solutions. Antigen retrieval was done by heating the slides for $15 \mathrm{~min}$ in a microwave oven in 10 $\mathrm{mM}$ citrate buffer ( $\mathrm{pH}$ 6.0). Endogenous peroxidase activity was blocked by incubation in 3\% hydrogen peroxide in TBS for 10 min, then the sections were incubated overnight at $4{ }^{\circ} \mathrm{C}$ in $1: 300$ dilutions of anti-RIP3 antibody. Chromogen was developed for 5 min with liquid 3,3'-diaminobenzidine (TL-015-HD, Thermo Scientific, Bioanalytica, Greece) and counterstained with Meyer's hematoxylin. Immunohistochemical staining was evaluated based on the proportion of stained cells and immunostaining intensity. $\mathrm{H}$-score was obtained by multiplying the proportion of stained cells (\%) and staining intensity graded 0 (negative), 1 (weak), 2 (moderate), or 3 (strong). H-score ranged from 0 to 300. Staining was carried out for tumor and normal tissue for each sample at the same time. Staining was interpreted by an experienced pathologist blinded to the clinical data (Ja Seung Koo). TUNEL staining was performed following the manufacturer's protocol (Chemicon, Temecula, CA, USA).

\section{Breast cancer subtyping}

Breast cancer phenotypes were classified according to the immunohistochemistry results for ER, PR, HER-2, and Ki-67 labeling index and FISH results for HER-2 according to the guidelines of Goldhirsch et al. [46]. Classification was as follows: luminal A type: PR and/or ER positive, HER-2 negative, and Ki-67 LI $<14 \%$; luminal B type: (HER-2 negative) PR and/or ER positive, HER-2 negative, and Ki-67 LI $\geq 14 \%$ and (HER-2 positive) PR and/or ER positive and HER-2 overexpressed and/or amplified; HER-2 type: PR and ER negative and HER-2 overexpressed and/ or amplified; TNBC type: PR, ER, and HER-2 negative.

\section{Kaplan-Meier analysis}

Kaplan-Meier analysis was done using the Breast Cancer GeneExpression Miner v3.0 software designed by Jézéquel et al. [36], without respect to nodal status or ER status. Data were pooled from studies in references [47-52].

\section{AML methylation and survival analysis}

Methylation analysis and survival analysis for AML patients was conducted for RIP3 using the methodology and data sets of Marcucci et al. [53], with promoter methylation analysis defined as $1 \mathrm{~kb}$ upstream and $1 \mathrm{~kb}$ downstream of TSS.

\section{Tumor xenograft study}

Animal care experimental procedures were conducted in accordance with the approval of the Inha Institutional Animal Care and Use Committee (INHA IACUC) at the Medical School of Inha University (INHA 131219-256). MDA-MB-231 cells were mixed with PBS and Matrigel $(200 \mu \mathrm{l} / \mathrm{mouse}$; suspended in $100 \mathrm{ml}$ of PBS mixed with $100 \mathrm{ml}$ of Matrigel), and orthotopically injected into mammary fat pads of 6-week-old BALB/c nu/nu female mice (Central Laboratory, Animal Inc, Seoul, Korea). Mice were fed with standard rat chow and tap water ad libitum, and maintained under a $12 \mathrm{~h}$ dark/light cycle at $21{ }^{\circ} \mathrm{C}$. When the tumors had reached a volume of about $80-100 \mathrm{~mm}^{3}$, mice were randomly assigned into four groups: untreated, treatment with 5-AD (1 mg/ $\mathrm{kg}$ by intraperitoneal injection four times for the first week and then $0.1 \mathrm{mg} / \mathrm{kg}$ by intraperitoneal injection five times/week) and/ or, doxorubicin (1 $\mathrm{mg} / \mathrm{kg}$ by intraperitoneal injection three times/ week for the second to third weeks, and then $2 \mathrm{mg} / \mathrm{kg}$ to the last day). Tumor dimensions were measured twice a week using a digital caliper and tumor volume was calculated using the formula $(\mathrm{V}$ $=$ length $\times$ width $\left.^{2} \times 0.5\right)$. Following the experiment, the mice were sacrificed and the tumors were excised and weighed.

\section{Statistical analysis}

Statistical analysis was performed using ANOVA and an unpaired Student's $t$-test. A $P$-value of 0.01 was considered statis- 
tically significant. Statistical calculations were performed using SPSS software for Windows Version 12.0 (SPSS, Chicago, IL, USA).

\section{Acknowledgments}

We thank Jiahuai Han for RIP3 $3^{-/-}$MEFs. This work was supported by the Basic Science Research Program of the National Research Foundation of Korea (NRF) and Ministry of Education, Science, and Technology of Korea (2011-0030043 and 2014R1A2A1A11052951) to Y-S Kim.

\section{References}

1 Hanahan D, Weinberg RA. Hallmarks of cancer: the next generation. Cell 2011; 144:646-674.

2 Vandenabeele P, Galluzzi L, Vanden Berghe T, Kroemer G. Molecular mechanisms of necroptosis: an ordered cellular explosion. Nat Rev Mol Cell Biol 2010; 11:700-714.

3 Vanlangenakker N, Vanden Berghe T, Vandenabeele P. Many stimuli pull the necrotic trigger, an overview. Cell Death Differ 2012; 19:75-86.

4 Morgan M, Liu Z. Programmed cell death with a necrotic-like phenotype. BioMol Concepts 2013; 4:259-275.

5 Kaiser WJ, Upton JW, Long AB, et al. RIP3 mediates the embryonic lethality of caspase-8-deficient mice. Nature 2011; 471:368-372.

6 Oberst A, Dillon CP, Weinlich R, et al. Catalytic activity of the caspase-8-FLIP(L) complex inhibits RIPK3-dependent necrosis. Nature 2011; 471:363-367.

7 Zhang H, Zhou X, McQuade T, Li J, Chan FK, Zhang J. Functional complementation between FADD and RIP1 in embryos and lymphocytes. Nature 2011; 471:373-376.

$8 \mathrm{Lu} \mathrm{JV}$, Weist BM, van Raam BJ, et al. Complementary roles of Fas-associated death domain (FADD) and receptor interacting protein kinase-3 (RIPK3) in T-cell homeostasis and antiviral immunity. Proc Natl Acad Sci USA 2011; 108:1531215317.

9 Bonnet MC, Preukschat D, Welz PS, et al. The adaptor protein FADD protects epidermal keratinocytes from necroptosis in vivo and prevents skin inflammation. Immunity 2011; 35:572-582.

10 Gunther C, Martini E, Wittkopf N, et al. Caspase-8 regulates TNF-alpha-induced epithelial necroptosis and terminal ileitis. Nature 2011; 477:335-339.

11 Welz PS, Wullaert A, Vlantis K, et al. FADD prevents RIP3-mediated epithelial cell necrosis and chronic intestinal inflammation. Nature 2011; 477:330-334.

12 Moriwaki K, Chan FK. RIP3: a molecular switch for necrosis and inflammation. Genes Dev 2013; 27:1640-1649.

13 Linkermann A, Brasen JH, Darding M, et al. Two independent pathways of regulated necrosis mediate ischemia-reperfusion injury. Proc Natl Acad Sci USA 2013; 110:12024-12029.

14 Kaiser WJ, Upton JW, Mocarski ES. Viral modulation of programmed necrosis. Curr Opin Virol 2013; 3:296-306.

15 Zhang DW, Shao J, Lin J, et al. RIP3, an energy metabolism regulator that switches TNF-induced cell death from apoptosis to necrosis. Science 2009; 325:332-336.
16 He S, Wang L, Miao L, et al. Receptor interacting protein kinase-3 determines cellular necrotic response to TNF-alpha. Cell 2009; 137:1100-1111.

17 Cho YS, Challa S, Moquin D, et al. Phosphorylation-driven assembly of the RIP1-RIP3 complex regulates programmed necrosis and virus-induced inflammation. Cell 2009; 137:1112-1123.

18 Zhao J, Jitkaew S, Cai Z, et al. Mixed lineage kinase domain-like is a key receptor interacting protein 3 downstream component of TNF-induced necrosis. Proc Natl Acad Sci USA 2012; 109:5322-5327.

19 Sun L, Wang H, Wang Z, et al. Mixed lineage kinase domain-like protein mediates necrosis signaling downstream of RIP3 kinase. Cell 2012; 148:213-227.

20 Wu J, Huang Z, Ren J, et al. Mlkl knockout mice demonstrate the indispensable role of Mlkl in necroptosis. Cell Res 2013; 23:994-1006.

21 Murphy JM, Czabotar PE, Hildebrand JM, et al. The pseudokinase MLKL mediates necroptosis via a molecular switch mechanism. Immunity 2013; 39:443-453.

22 Cai Z, Jitkaew S, Zhao J, et al. Plasma membrane translocation of trimerized MLKL protein is required for TNF-induced necroptosis. Nat Cell Biol 2014; 16:55-65.

23 Chen X, Li W, Ren J, et al. Translocation of mixed lineage kinase domain-like protein to plasma membrane leads to necrotic cell death. Cell Res 2014; 24:105-121.

24 Wang H, Sun L, Su L, et al. Mixed lineage kinase domainlike protein MLKL causes necrotic membrane disruption upon phosphorylation by RIP3. Mol Cell 2014; 54:133-146.

25 Dondelinger Y, Declercq W, Montessuit S, et al. MLKL compromises plasma membrane integrity by binding to phosphatidylinositol phosphates. Cell Rep 2014; 7:971-981.

26 Tenev T, Bianchi K, Darding M, et al. The ripoptosome, a signaling platform that assembles in response to genotoxic stress and loss of IAPs. Mol Cell 2011; 43:432-448.

27 Dondelinger Y, Aguileta MA, Goossens V, et al. RIPK3 contributes to TNFR1-mediated RIPK1 kinase-dependent apoptosis in conditions of cIAP1/2 depletion or TAK1 kinase inhibition. Cell Death Differ 2013; 20:1381-1392.

28 Cook WD, Moujalled DM, Ralph TJ, et al. RIPK1- and RIPK3-induced cell death mode is determined by target availability. Cell Death Differ 2014; 21:1600-1612.

29 Kim YS, Morgan MJ, Choksi S, Liu ZG. TNF-induced activation of the Nox1 NADPH oxidase and its role in the induction of necrotic cell death. Mol Cell 2007; 26:675-687.

30 Ventura JJ, Cogswell P, Flavell RA, Baldwin AS Jr, Davis RJ. JNK potentiates TNF-stimulated necrosis by increasing the production of cytotoxic reactive oxygen species. Genes Dev 2004; 18:2905-2915.

31 Degterev A, Hitomi J, Germscheid M, et al. Identification of RIP1 kinase as a specific cellular target of necrostatins. Nat Chem Biol 2008; 4:313-321.

32 Li JX, Feng JM, Wang Y, et al. The B-Raf(V600E) inhibitor dabrafenib selectively inhibits RIP3 and alleviates acetaminophen-induced liver injury. Cell Death Dis 2014; 5:e1278.

33 Veeck J, Noetzel E, Bektas N, et al. Promoter hypermethylation of the SFRP2 gene is a high-frequent alteration and tumor-specific epigenetic marker in human breast cancer. Mol Cancer 2008; 7:83. 
34 Irizarry RA, Ladd-Acosta C, Wen B, et al. The human colon cancer methylome shows similar hypo- and hypermethylation at conserved tissue-specific $\mathrm{CpG}$ island shores. Nat Genet 2009; 41:178-186.

35 Ghoshal K, Datta J, Majumder S, et al. 5-Aza-deoxycytidine induces selective degradation of DNA methyltransferase 1 by a proteasomal pathway that requires the KEN box, bromo-adjacent homology domain, and nuclear localization signal. Mol Cell Biol 2005; 25:4727-4741.

36 Jezequel P, Campone M, Gouraud W, et al. bc-GenExMiner: an easy-to-use online platform for gene prognostic analyses in breast cancer. Breast Cancer Res Treat 2012; 131:765-775.

37 Biton S, Ashkenazi A. NEMO and RIP1 control cell fate in response to extensive DNA damage via TNF- $\alpha$ feedforward signaling. Cell 2011; 145:92-103.

38 Feoktistova M, Geserick P, Kellert B, et al. cIAPs block ripoptosome formation, a RIP1/caspase- 8 containing intracellular cell death complex differentially regulated by cFLIP isoforms. Mol Cell 2011; 43:449-463.

39 Micheau O, Tschopp J. Induction of TNF receptor I-mediated apoptosis via two sequential signaling complexes. Cell 2003; 114:181-190.

40 Zong WX, Ditsworth D, Bauer DE, Wang ZQ, Thompson CB. Alkylating DNA damage stimulates a regulated form of necrotic cell death. Genes Dev 2004; 18:1272-1282.

41 Sosna J, Voigt S, Mathieu S, et al. TNF-induced necroptosis and PARP-1-mediated necrosis represent distinct routes to programmed necrotic cell death. Cell Mol Life Sci 2014; 71:331-348.

42 Kasof GM, Prosser JC, Liu DR, Lorenzi MV, Gomes BC. The RIP-like kinase, RIP3, induces apoptosis and NF- $\mathrm{B}$ nuclear translocation and localizes to mitochondria. FEBS Lett 2000; 473:285-291.

43 Newton K, Sun XQ, Dixit VM. Kinase RIP3 is dispensable for normal NF- $\kappa \mathrm{Bs}$, signaling by the B-cell and T-cell receptors, tumor necrosis factor receptor 1 , and toll-like receptors 2 and 4. Mol Cell Biol 2004; 24:1464-1469.

44 Sun XQ, Lee J, Navas T, Baldwin DT, Stewart TA, Dixit VM. RIP3, a novel apoptosis-inducing kinase. J Biol Chem 1999; 274:16871-16875.

45 Cowan LA, Talwar S, Yang AS. Will DNA methylation inhibitors work in solid tumors? A review of the clinical experience with azacitidine and decitabine in solid tumors. Epigenomics 2010; 2:71-86.

46 Goldhirsch A, Wood WC, Coates AS, et al. Strategies for subtypes-dealing with the diversity of breast cancer: highlights of the St Gallen International expert consensus on the primary therapy of early breast cancer 2011. Ann Oncol 2011; 22:1736-1747.

47 van de Vijver MJ, He YD, van't Veer LJ, et al. A gene-expression signature as a predictor of survival in breast cancer. $N$ Engl J Med 2002; 347:1999-2009.

48 Pawitan Y, Bjohle J, Amler L, et al. Gene expression profiling spares early breast cancer patients from adjuvant therapy: derived and validated in two population-based cohorts. Breast Cancer Res 2005; 7:R953-R964.

49 Weigelt B, Hu Z, He X, et al. Molecular portraits and 70-gene prognosis signature are preserved throughout the metastatic process of breast cancer. Cancer Res 2005; 65:9155-9158.

50 Loi S, Haibe-Kains B, Desmedt C, et al. Definition of clinically distinct molecular subtypes in estrogen receptor-positive breast carcinomas through genomic grade. J Clin Oncol 2007; 25:1239-1246.

51 Chanrion M, Negre V, Fontaine H, et al. A gene expression signature that can predict the recurrence of tamoxifen-treated primary breast cancer. Clin Cancer Res 2008; 14:1744-1752.

52 Loi S, Haibe-Kains B, Desmedt C, et al. Predicting prognosis using molecular profiling in estrogen receptor-positive breast cancer treated with tamoxifen. BMC Genomics 2008; 9:239.

53 Marcucci G, Yan P, Maharry K, et al. Epigenetics meets genetics in acute myeloid leukemia: clinical impact of a novel seven-gene score. J Clin Oncol 2014; 32:548-556.

(Supplementary information is linked to the online version of the paper on the Cell Research website.) 